\title{
Modeling and Analysis of Channel Holding Time and Handoff Rate for Packet Sessions in All-IP Cellular Networks
}

\author{
Bying-He Ku, Yi Ren, Member, IEEE, Jeng-Feng Weng, Member, IEEE, \\ Jyh-Cheng Chen, Fellow, IEEE, and Wen-Tsuen Chen, Fellow, IEEE
}

\begin{abstract}
It is essential to model channel holding time (CHT), cell residence time (CRT), and handoff rate for performance analysis and algorithm evaluation in mobile cellular networks. The problem has been extensively studied in the past for circuitswitched (CS) cellular networks. However, little research has been done on packet-switched (PS) cellular networks. Unlike that a call occupies a dedicated channel during its whole lifetime in CS networks, an active session in PS networks occupies and releases channels iteratively due to discontinuous reception (DRX) mechanism. In this paper, we investigate the key quantities in PS cellular networks. We present a set of comprehensive new models to characterize the quantities and their relationship in PS networks. The models shed light on the relationship between CHT and CRT and handoff rate. The analytical results enable wide applicability in various scenarios and therefore have important theoretical significance. Moreover, the analytical results provide a quick way to evaluate traffic performance and system design in PS cellular networks without wide deployment, which can save cost and time.
\end{abstract}

Index Terms-Cell residence time (CRT), channel holding time (CHT), handoff rate, mobility, modeling and analysis, packet session.

\section{INTRODUCTION}

$\mathbf{M}$ OBILE cellular networks have become one of the essential requirements in our daily life. A recent study from Ericsson reports that in Q3 of 2015, the total mobile subscriptions and global mobile penetration rate have reached 7.3 billion and $95 \%$, respectively [1]. Mobile services are moving from the traditional circuit-switched (CS) toward packet-switched (PS), such as VoLTE or voice over Long-Term Evolution (LTE).

The modeling of user equipment (UE) mobility, which can characterize mobile cellular network dynamics with appropriate accuracy, has wide usage in network planning, resource

Manuscript received January 2, 2016; revised April 3, 2016; accepted June 13, 2016. Date of publication July 7, 2016; date of current version April 14, 2017. This work was supported in part by the Ministry of Science and Technology of Taiwan under Grant MOST 103-2221-E-009-110-MY3, Grant MOST 104-3115-E-009-006, Grant MOST 103-2221-E-001-018, and Grant MOST 104-2221-E-001-002; by the Hsinchu Science Park Bureau under Grant 104A08; and by the Thematic Research Grant, Academia Sinica, Taiwan. The review of this paper was coordinated by Dr. P. Lin.

B.-H. Ku and W.-T. Chen are with the Institute of Information Science, Academia Sinica, Taipei 115, Taiwan (e-mail: byinghe.ku@gmail.com; wtchen@cs. nthu.edu.tw).

Y. Ren, J.-F. Weng, and J.-C. Chen are with the Department of Computer Science, National Chiao Tung University, Hsinchu 300, Taiwan (e-mail: renyi@ cs.nctu.edu.tw; jcc@cs.nctu.edu.tw; jengfeng.weng@gmail.com).

Color versions of one or more of the figures in this paper are available online at http://ieeexplore.ieee.org. optimization, network performance analysis, and new service evaluations. With UE mobility models, mobile operators can quickly obtain the performance of their new system designs (e.g., tracking area and location update area) and evaluate new algorithms to reduce handoff cost without wide implementation and deployment. It can save cost and time.

Channel holding time (CHT), cell residence time (CRT), and handoff rate are key quantities for UE mobility modeling. These quantities are highly related. Specifically, CHT allows one to derive key network parameters such as new call/session blocking probability, handoff call/session blocking probability [2], etc. It has been widely used for the evaluation of the mobile routing strategy [3] and resource allocation [4], and models for a data charging system [5], spectrum sensing [6], and minimizing unnecessary handoffs [7], [8]. The CHT depends on UE mobility, which can be characterized by CRT, which is determined by cell size, UE speed, and movement direction. The handoff rate is also related to CHT. It is defined as the mean number of handoffs during an active call and one of the key quantities to model CHT distribution. Therefore, it is important to find an appropriate model to characterize these quantities and their relationship, which is the focus of this paper.

In CS cellular networks, extensive studies have been carried out for handoff performance in terms of CHT, CRT, and handoff rate [6]-[26]. For the sake of convenience and analytical tractability, a common assumption in these studies is that the CHT and/or CRT is exponentially distributed. Hong and Rappaport in [9] proposed the first traffic model for cellular mobile telephone networks, indicating that CHT could be approximated by an exponential distribution when CRT is exponentially distributed. Thereafter, plenty of research studies (e.g., [21]-[23]) have been conducted under the exponential distribution assumption. Recent studies [17], [18] have addressed that exponential distribution cannot capture the overall effects of UE mobility. Instead, the use of general distributions to model such quantities has been proposed, such as Erlang [11], [15], [17], Gamma [20], Uniform [9], deterministic [26], hyper-Erlang [15], [19], lognormal [25], Pareto [24], Weibull distribution [18], and sum of hyperexponentials [10].

However, the work [6]-[26] developed for CS cellular networks cannot be applied to PS cellular networks directly, due to the following reasons.

- Unlike CS networks in that an active call holds its channel during its whole lifetime, a packet session in the PS 

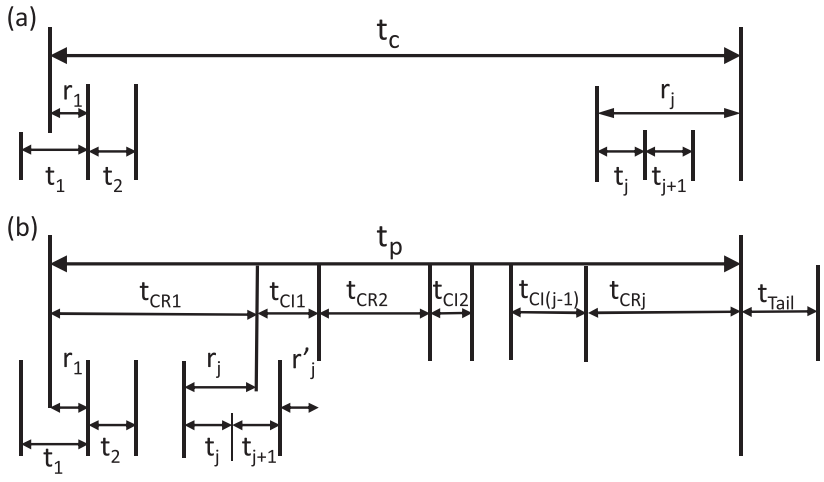

Fig. 1. (a) Timing diagram for CS cellular networks. (b) Timing diagram for PS cellular networks.

network occupies and releases its channel iteratively for the duration of the packet session to save UE energy.

- An active call in the CS network releases its channel when it is terminated. However, in the PS network, the UE does not release Radio Resource Control (RRC) connection until there is no data transmission for an extended period of time.

Fig. 1(a) shows the timing diagram for the CHT (denoted as $t_{c}$ ) in CS cellular networks. Specifically, in traditional CS networks, voice traffic is transmitted in a dedicated channel in which the resource is not released until the call connection terminates. However, during a packet session, the UE may sleep in some periods and partially release its resources. In comparison, Fig. 1(b) shows the timing diagram for PS cellular networks. $t_{p}$ denotes the time interval between the time data transmission starts until the time it terminates. During $t_{p}$, the UE occupies the channel in $t_{C R 1}, t_{C R 2}, \ldots, t_{C R j}$, and sleeps in $t_{C I 1}$, $t_{C I 2}, \ldots, t_{C I j-1}$. Moreover, even if there is no packet transmission after $t_{p}$, the resources are still held for the UE during an extended time interval $t_{\text {Tail }}$. Considering the discontinuous reception (DRX) in LTE as an example, the UE turns on its receiver at certain predefined time points and sleeps at other periods (will be discussed in Section II-B) to save energy. During the sleep periods, the resource (i.e., LTE-U_u interface [27]) between the UE and the evolved Node B (eNB) is released. The differences between CS and PS networks motivate us to study UE mobility modeling for packet sessions. More details about Fig. 1 will be discussed later.

In this paper, we investigate the modeling of CHT, CRT, and handoff rate in PS cellular networks. As in [11], [13], [15], [19], [28], and [29], CHT depends on the user's mobility, which is characterized by CRT. We further define an effect CHT (eCHT) to model the extended resource holding time for UE devices in $t_{C I j}$ and $t_{\text {Tail }}$, where the eCHT experiences active and idle periods in a session. We are the first to introduce eCHT and address the relationship between eCHT and CRT in packet sessions. Based on our analytical results, their relationship is as follows.

- If CRT is exponentially distributed, eCHT is exponentially distributed as well, and vice versa.

- Again, if CRT is exponentially distributed, the new session's eCHT and the handoff session's eCHT will have the same distribution, and vice versa.
These results provide critical guidelines for UE mobility modeling and can prevent researchers from making inappropriate assumptions on eCHT and CRT, e.g., assuming eCHT and CRT as an Erlang distribution and an exponential distribution, respectively. Moreover, we demonstrate that the quantity of handoff rate is not only used in characterizing the eCHT distribution but also to be tied to the service quality and cost parameters. We derive a general formula for the computation of the handoff rate under general conditions. The results enable wide applicability in various scenarios and therefore have important theoretical significance. The models presented in this paper can be used as a theoretical guideline for network designers and performance analysts.

The rest of this paper is organized as follows. In Section II, we introduce the hyper-Erlang distribution and review the background of DRX mode in LTE. Section III presents a new mathematical model to analyze CRT, (conditional) CHT with DRX mode, and handoff rate in a packet session. Numerical results are shown in Section IV. Section V offers conclusions.

\section{PRELIMINARIES AND BACKGROUND}

Here, we first briefly introduce the Erlang distribution and the hyper-Erlang distribution and then present the background of the LTE DRX mechanism.

\section{A. Erlang Distribution and Hyper-Erlang Distribution}

Erlang distribution has the following probability density function (pdf) and Laplace transform (LT):

$$
f(t)=\frac{\beta^{m} t^{m-1}}{(m-1) !} e^{-\beta t}, f^{*}(s)=\left(\frac{\beta}{s+\beta}\right)^{m} .
$$

Note that when $m=1, f(t)$ is an exponential distribution.

According to the work in [11], [15], and [19], the pdf and LT of the hyper-Erlang distribution with $M$ parameters are as follows:

$$
\begin{aligned}
& f_{h e}(t)=\sum_{i=1}^{M} \alpha_{i} \frac{\left(m_{i} \eta_{i}\right)^{m_{i}} t^{m_{i}-1}}{\left(m_{i}-1\right) !} e^{-m_{i} \eta_{i} t}, t \geq 0 \\
& f_{\text {he }}^{*}(s)=\sum_{i=1}^{M} \alpha_{i}\left(\frac{m_{i} \eta_{i}}{s+m_{i} \eta_{i}}\right)^{m_{i}} .
\end{aligned}
$$

Note that when $M=1$ (i.e., hyper-Erlang distribution with one parameter), $f_{h e}(t)$ is an Erlang distribution.

A hyper-Erlang distribution model with two parameters is used in our numerical study, where hyper-Erlang distribution with two parameters is as follows:

$$
f^{*}(s)=\alpha_{1}\left(\frac{m_{1} \eta}{s+m_{1} \eta}\right)^{m_{1}}+\alpha_{2}\left(\frac{m_{2} \eta}{s+m_{2} \eta}\right)^{m_{2}} .
$$

\section{B. DRX Mode in LTE}

The 3rd Generation Partnership Project has defined DRX [27] to increase the UE's battery lifetime and resource utilization and to minimize packet delay. With DRX, the UE 


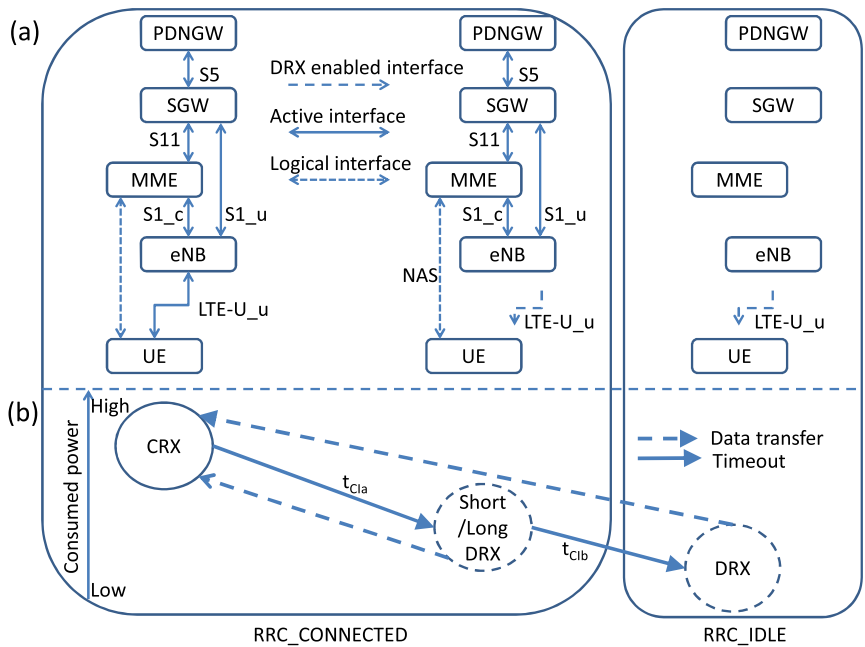

Fig. 2. DRX in LTE.

turns on the receiver at some predefined time points and sleeps at other time periods. The UE periodically wakes up to listen to the downlink (DL) transmissions, following the DRX cycle.

Fig. 2(a) shows the network architecture in which the Evolved Packet System (EPS) network interfaces are depicted. When a UE device is in DRX enabled/RRC_IDLE state, the eNB may initiate the release of the UE's RRC connection and request the Mobility Management Entity (MME) to release the UE's S1 connection, i.e., the S1, NAS, and RRC connections are removed.

The DRX state machine in LTE is shown in Fig. 2(b), in which a UE device has two different modes: RRC_CONNECTED and RRC_IDLE. In the RRC_CONNECTED state, the UE moves from continuous reception (CRX) to short DRX state and then shifts to long DRX state when the UE/eNB is waiting for data packets for an extended period after the last transmitted/ received packet (i.e., DRX inactivity timer expires [30]). If the channel is idle longer than the predefined DRX inactivity timer, which is denoted as $a$, the RRC will then move from RRC CONNECTED to RRC_IDLE [31].

From the viewpoint of holding resource in this paper, we consider the eCHT when a UE device is in the RRC CONNECTED state. Even in RRC_CONNECTED, a UE device may still sleep in long/short DRX state.

\section{Modeling CHT, CRT, And Handoff RATE IN PS CELlular NeTwORKS}

Here, we propose analytical models for UE mobility modeling in PS cellular networks. Moreover, we derive the general formula for handoff rates. The model of handoff rate can be used to characterize handoff session arrival rate, eCHT distribution, service quality, and cost parameters.

Section III-A extends the work in [11] to PS networks and proves that its results are also valid in PS networks, i.e., the eCHTs are exponentially distributed if and only if the CRTs are exponentially distributed. This result reveals the fundamental relationship between eCHT and CRT. Section III-B relaxes the distribution assumption of CRT (i.e., the CRTs are not exponentially distributed) to derive the eCHT distribution. It provides a very general model for UE mobility modeling in PS networks. Later, Section III-C derives the model of conditional eCHT and shows its opposite results compared with the model in a CS network. Finally, another important quantity (handoff rate) for eCHT modeling is presented in Section III-D.

\section{A. Modeling eCHT for Exponentially Distributed CRT}

Here, we present our analytical model for eCHT considering that CRT is exponentially distributed. Recall that in the RRC_CONNECTED state, a UE device changes between active and sleep modes from time to time. We denote them as CRX time $t_{C R j}(j=1,2, \ldots)$ and Connected-Idle time $t_{C I_{j}}$ $(j=1,2, \ldots)$, respectively, as shown in Fig. 1(b). During CRX time, i.e., $t_{C R j}$, the channel is used for packet transmission. If there is no packet transmission, the UE enters sleep mode, i.e., $t_{C I j}(j=1,2, \ldots)$. Once there is a packet arrival, the UE changes to $t_{C R j}$. The $t_{C R j}$ corresponds to the CRX state in Fig. 2(b). The $t_{C I j}$ includes short DRX and long DRX, in which the channel is idle. The maximum idle time is denoted as $t_{\text {Tail }}$. If the idle time of the channel is longer than $t_{\text {Tail }}$, the UE enters RRC_IDLE mode, and we consider that the packet session is finished.

During $t_{C I j}$, a UE device has no activity in both DL (forward link) and uplink (reverse link). The maximum value of $t_{C I j}$ is the length of the idle timer $t_{\text {Tail }}$. Let $r_{1}$ be the period between the time a new packet session starts and the time the session leaves the serving cell but is not finished yet. Let $t_{j}$ be the CRT and $r_{j}(j \geq 2)$ be the residual lifetime of the eCHT after the packet session completes the $j-1$ th handoff successfully.

Let $t_{e}$ represent the $e C H T$. We then can obtain

$$
t_{e}=t_{p}+t_{\text {Tail }}, t_{C I j}>t_{\text {Tail }} .
$$

In Fig. 1(b), we have the following four eCHTs.

1) $t_{p n h}$ : The new session eCHT [15], [16] is shown in Fig. 3(a), i.e.,

$$
t_{p n h}=\min \left\{t_{p}+t_{\text {Tail }}, r_{1}\right\} .
$$

Specifically, there are two cases for $t_{p n h}$ : 1) A new session starts at point A and hands off to another cell at point B. For this case, $\left.t_{p n h}=r_{1} ; 2\right)$ a new session initiates at $\mathrm{C}$ and terminates at $\mathrm{D}$, i.e., $t_{p n h}=t_{p}+t_{\text {Tail }}$. Here, we assume that $\lambda$ is the new session arrival rate to a cell.

2) $t_{\text {phh_busy }}$ : The handoff session $e C H T$ in busy mode [15], [16] is shown in Fig. 3(b), i.e.,

$$
t_{p h h_{-} \text {busy }}=\min \left\{r_{j}+t_{\text {Tail }}, t_{j}\right\} .
$$

Here, the busy mode means that the UE is in CRX state when the handoff is conducted. Similarly, the session either hands off to another cell at point B or terminates at $\mathrm{D}$. We assume that $\lambda_{h b}$ is the arrival rate of handoff sessions in busy mode. 
(a)

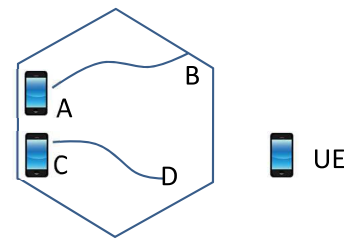

(c)

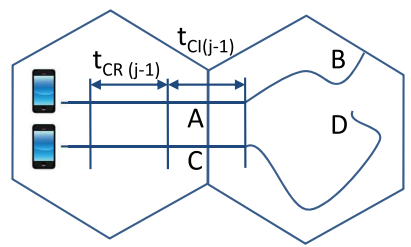

(b)

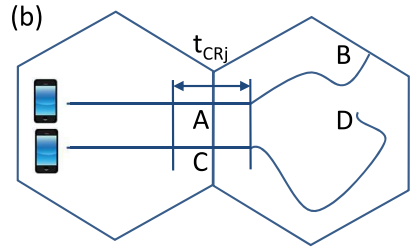

(d)

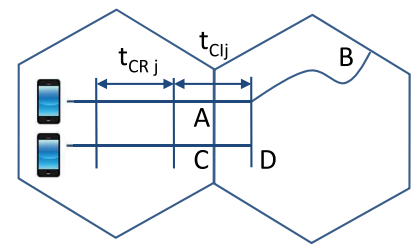

Fig. 3. (a) $\mathrm{CRT}\left(\overline{A B} \equiv r_{1}\right)$ and residual lifetime $\left(\overline{C D} \equiv t_{p}+t_{\text {Tail }}\right)$ of a new session, (b) that of a handoff session in busy mode with a new session, (c) that of a handoff session in idle mode with a new session, and (d) that of a handoff session in idle mode without new sessions.

3) $t_{p h h_{-} \text {idle_new }}$ Fig. 3(c) shows that the handoff session $e C H T$ is in idle mode and then enters busy mode, i.e.,

$$
t_{p h L_{-} \text {idle_new }}=\min \left\{r_{j}+t_{\text {Tail }}, t_{j}\right\} .
$$

Here, the idle mode means that the UE is in short/long DRX state when the handoff is conducted. We assume that $\lambda_{h d a}$ is the arrival rate of handoff sessions in idle mode. Discussion: An interesting observation is that $t_{p h h_{\text {busy }}}$ and $t_{p h h_{\text {_idle_new }}}$ have the same formulas, i.e., (5) is the same as (6). The reasons are as follows. For the handoff session in busy mode, as shown in Fig. 3(b), when the UE completes the data transmission in the right cell, the UE then changes to idle mode. In Fig. 3(c), the UE shifts from idle mode to busy mode again in the right cell. Because $r_{j}$ is the residual lifetime after the $j$ th successful handoff and the user will finally be in long DRX mode (i.e., the maximum value of $t_{C I j}=t_{\text {Tail }}$ ), we have $(5) \equiv(6)$.

4) $t_{p h h_{\text {iddle_no }}}$ : The handoff session $e C H T$ with no arrival packet in idle mode is shown in Fig. 3(d), i.e.,

$$
t_{p h{ }_{\text {_idle_no }}}=\min \left\{r_{j}^{\prime}+t_{\text {Tail }}, t_{j}\right\} .
$$

We assume that $\lambda_{h d b}$ is the arrival rate of handoff sessions in idle mode.

Here, we define pdfs $f_{p}(t), f_{C I j}(t), f_{d}(t), f_{r}(t), f_{p n h}(t)$, $f_{p h h_{-} \text {busy }}(t), f_{p h h_{-} \text {idle_new }}(t)$, and $f_{p h h_{-} \text {idle_no }}(t)$; cumulative distribution functions (cdfs) $F_{p}(t), F_{C I j}(t), F_{d}(t), F_{r}(t)$, $F_{p n h}(t), F_{p h h_{-} \text {busy }}(t), F_{p h h_{-} \text {idle_new }}(t)$, and $F_{p h h_{-} \text {idle_no }}(t)$; and LTs $f_{p}^{*}(s), f_{C I j}^{*}(s), f_{d}^{*}(s), f_{r}^{*}(s), f_{p n h}^{*}(s), f_{p h h_{-} \text {busy }}^{*}(s)$, $f_{p h h_{-} \text {idle_new }}^{*}(s)$, and $f_{p h h_{\text {_idle_no }}}^{*}(s)$ for $t_{p}, t_{C I j}, t_{j}, r_{1}, t_{p n h}$, $t_{p h h_{-} \text {busy }}, t_{p h h_{-} \text {idle_new }}$, and $t_{p h h_{-} \text {idle_no, }}$ respectively (see Table I for details).

In the following, we will answer the question of how to model the eCHT for $t_{p n h}, t_{p h h_{-} \text {busy }}, t_{p h h_{-} \text {idle_new }}$, and

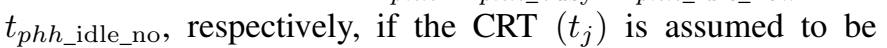
exponentially distributed.

1) Handoff Session in Busy Mode: First, we can obtain the conditional probability of (5) as

$$
\begin{gathered}
P\left(t_{\text {phh_busy }} \leq t \mid t_{C I j}>t_{\text {Tail }}\right) \\
=P\left(r_{j}+t_{\text {Tail }} \leq t \text { or } t_{j} \leq t \mid t_{C I j}>t_{\text {Tail }}\right) \\
=P\left(\left(r_{j}+t_{\text {Tail }}\right) \leq t \mid t_{C I j}>t_{\text {Tail }}\right) \\
\quad+P\left(t_{j} \leq t \mid t_{C I j}>t_{\text {Tail }}\right) \\
\quad-P\left(\left(\left(r_{j}+t_{\text {Tail }}\right) \leq t, t_{j} \leq t\right) \mid t_{C I j}>t_{\text {Tail }}\right) \\
=P\left(\left(r_{j}+t_{\text {Tail }}\right) \leq t\right)+P\left(t_{j} \leq t\right) \\
\quad-P\left(\left(r_{j}+t_{\text {Tail }}\right) \leq t\right) P\left(t_{j} \leq t\right) \\
=P\left(t_{p} \leq t-t_{\text {Tail }}\right)+P\left(t_{j} \leq t\right) \\
\quad-P\left(t_{p} \leq t-t_{\text {Tail }}\right) P\left(t_{j} \leq t\right)
\end{gathered}
$$

where we have $P\left(r_{j}+t_{\text {Tail }} \leq t, t_{j} \leq t\right)=P\left(r_{j}+t_{\text {Tail }} \leq t\right)$ $P\left(t_{j} \leq t\right)$ from the independence of $r_{j}, t_{j}$, and $t_{C I j}$ [11], [15], [16]. From the Residual Life Theorem [32] or the argument in [33], we also have $P\left(r_{j}+t_{\text {Tail }} \leq t\right)=P\left(t_{p} \leq t-t_{\text {Tail }}\right)$ based on the memoryless property of the exponential distribution, where $r_{j}$ has the same distribution as $t_{p}$.

By differentiating (8), we have the following conditional pdf:

$$
\begin{aligned}
f_{p h h_{\_} \text {busy }}(t)= & f_{p}\left(t-t_{\text {Tail }}\right)+f_{d}(t)-f_{p}\left(t-t_{\text {Tail }}\right) P\left(t_{j} \leq t\right) \\
& -P\left(t_{p} \leq t-t_{\text {Tail }}\right) f_{d}(t) \\
= & f_{p}\left(t-t_{\text {Tail }}\right)-f_{p}\left(t-t_{\text {Tail }}\right) \cdot \int_{0}^{t} f_{d}(\tau) d \tau \\
& +f_{d}(t)-f_{d}(t) \cdot \int_{0}^{t} f_{p}\left(\tau-t_{\text {Tail }}\right) d \tau
\end{aligned}
$$

Suppose that the CRTs $t_{j}$ are exponentially distributed with parameter $\eta$, then, from (9), we obtain

$$
\begin{aligned}
f_{p h h_{-} \text {busy }}(t) & =\mu e^{-\mu\left(t-t_{\text {Tail }}\right)} \times e^{-\eta t}+\eta e^{\eta t} \times e^{-\mu\left(t-t_{\text {Tail }}\right)} \\
& =(\mu+\eta) e^{-\left[\mu\left(t-t_{\text {Tail }}\right)+\eta t\right]} \\
& =(\mu+\eta) e^{-(\mu+\eta)\left(t-\frac{\mu t_{\text {Tail }}}{\mu+\eta}\right)}
\end{aligned}
$$

which is a two-parameter exponential distribution [34]. Here, we have shown that if the CRT is exponentially distributed, $t_{p h h_{-} \text {busy }}$, the handoff session eCHT in busy mode is exponentially distributed.

In contrast, suppose that $t_{p h h_{-} \text {busy }}$ is exponentially distributed with parameter $\gamma$. Let $Z(t)=\int_{t}^{\infty} f_{d}(\tau) d \tau$, then $Z(t)^{\prime}=$ $-f_{d}(t)$. From (9), we get

$$
\mu e^{-\mu\left(t-t_{\text {Tail }}\right)} Z(t)+e^{-\mu\left(t-t_{\text {Tail }}\right)} f_{d}(t)=\gamma e^{-\gamma \tau} .
$$

From (11), we can derive

$$
\begin{aligned}
Z(t) & =e^{\mu\left(t-t_{\text {Tail }}\right)} Z(0)+\int_{0}^{t} e^{\mu\left[\left(t-t_{\text {Tail }}\right)-\tau\right]}\left[-\gamma e^{-(\gamma-\mu) \tau}\right] d \tau \\
& =e^{\mu\left(t-t_{\text {Tail }}\right)}\left\{Z(0)-\gamma \int_{0}^{t} e^{-\gamma \tau} d \tau\right\} \\
& =e^{\mu t_{\text {Tail }}} \cdot e^{-(\gamma-\mu) t} .
\end{aligned}
$$


TABLE I

LIST OF PARAMETERS

\begin{tabular}{|c|c|c|c|c|}
\hline Variable & pdf & Laplace transform & $\mathrm{CDF}$ & Definition \\
\hline$t_{p}$ & $f_{p}(t)$ & $f_{p}^{*}(s)$ & $F_{p}(t)$ & Packet session CHT \\
\hline$t_{C I j}$ & $f_{C I j}(t)$ & $f_{C I j}^{*}(s)$ & $F_{C I j}(t)$ & Connected-Idle time, the maximum $t_{C I j}$ is $t_{T a i l}$ \\
\hline$t_{C R j}$ & & & & Continuous reception time (CRX) \\
\hline$r_{1}$ & $f_{r}(t)$ & $f_{r}^{*}(s)$ & $F_{r}(t)$ & Time period from a new session starts to leave the serving cell \\
\hline$r_{j}$ & & & & Residual life time after the $j^{t h}$ successful handoff \\
\hline$t_{j}$ & $f_{d}(t)$ & $f_{d}^{*}(s)$ & $F_{d}(t)$ & Cell Residence Time (CRT) \\
\hline$t_{p n h}$ & $f_{p n h}(t)$ & $f_{p n h}^{*}(s)$ & $F_{p n h}(t)$ & new session effective CHT (eCHT) \\
\hline$t_{\text {phh_busy }}$ & $f_{p h h_{-} b u s y}(t)$ & $f_{\text {phh_busy }}^{*}(s)$ & $F_{\text {phh_busy }}(t)$ & handoff session eCHT in busy mode \\
\hline$t_{p h h_{-} i d l e \_n e w}$ & $f_{p h h_{-} i d l e_{-} n e w}(t)$ & $f_{p h h_{-} i d l e_{-} n e w}^{*}(s)$ & $F_{p h h \_i d l e \_n e w}(t)$ & handoff session eCHT with new arrival packets in idle mode \\
\hline $\begin{array}{l}t_{p h h \_i d l e \_n o} \\
t_{c}\end{array}$ & $f_{p h h \_i d l e \_n o}(t)$ & $f_{p h h_{-} i d l e_{-} n o}^{*}(s)$ & $F_{p h h \_i d l e \_n o}(t)$ & $\begin{array}{l}\text { handoff session eCHT without arrival packet in idle mode } \\
\text { Circuit call CHT }\end{array}$ \\
\hline
\end{tabular}

Thus, $Z(t)^{\prime}=-(\gamma-\mu) e^{\left[-(\gamma-\mu) t+\mu t_{\text {Tail }}\right]}$. Finally, we obtain

$$
\begin{aligned}
f_{\text {phh_busy }}(t) & =(\gamma-\mu) e^{\left[-(\gamma-\mu) t+\mu t_{\text {Tail }}\right]} \\
& =(\gamma-\mu) e^{-(\gamma-\mu)\left(t-\frac{\mu t_{\text {Tail }}}{\gamma-\mu}\right)} .
\end{aligned}
$$

As a conclusion, (13) and (10) show a strong result that the handoff session eCHT in busy mode $t_{p h h_{-} \text {busy }}$ is exponentially distributed if the CRT is assumed to be exponentially distributed, and vice versa.

2) New Session: Similarly, the conditional probability of (4) is

$$
\begin{aligned}
& P\left(t_{\text {pnh }} \leq t \mid t_{C I j}>t_{\text {Tail }}\right) \\
& =P\left(t_{p}+t_{\text {Tail }} \leq t \text { or } r_{1} \leq t \mid t_{C I j}>t_{\text {Tail }}\right) \\
& =P\left(t_{p} \leq t-t_{\text {Tail }}\right)+P\left(r_{1} \leq t\right) \\
& \quad-P\left(t_{p} \leq t-t_{\text {Tail }}\right) P\left(r_{1} \leq t\right)
\end{aligned}
$$

By differentiating the given equation, we obtain

$$
\begin{aligned}
f_{\text {pnh }}(t)= & f_{p}\left(t-t_{\text {Tail }}\right)+f_{r}(t)-f_{p}\left(t-t_{\text {Tail }}\right) P\left(r_{1} \leq t\right) \\
& -P\left(t_{p} \leq t-t_{\text {Tail }}\right) f_{r}(t) \\
= & f_{p}\left(t-t_{\text {Tail }}\right)-f_{p}\left(t-t_{\text {Tail }}\right) \times \int_{0}^{t} f_{r}(\tau) d \tau+f_{r}(t) \\
& -f_{r}(t) \cdot \int_{0}^{t} f_{p}\left(\tau-t_{\text {Tail }}\right) d \tau .
\end{aligned}
$$

Suppose that the new session eCHT is exponentially distributed with parameter $\mu_{1}$. From a similar derivation of $t_{\text {phh_busy }}$, we can deduce that

$$
f_{r}(t)=\left(\mu_{1}-\mu\right) e^{-\left(\mu_{1}-\mu\right)\left(t-\frac{\mu t_{\text {Tail }}}{\mu_{1}-\mu}\right)}
$$

which is also an exponential distribution.

3) Handoff Session in Idle With New Arrival Packets: Recall that the handoff session eCHT with new arrival packets in idle mode, i.e., $t_{p h h \_i d l e \_n e w}$, is the same as the handoff session eCHT in busy mode, i.e., $t_{p h h_{-} \text {busy }}$. That is, $(5) \equiv(6)$. Based on Section III-A.1, we can derive that $t_{p h h \_i d l e \_n e w ~}$ is also exponentially distributed if and only if the CRT is an exponential distribution.
4) Handoff Session in Idle Without Arrival Packet: From (7), we get the following conditional probability:

$$
\begin{aligned}
& P\left(t_{p h h_{-} \text {idle_no }} \leq t \mid t_{C I j}>t_{\text {Tail }}\right) \\
& =P\left(r_{j}^{\prime}+t_{\text {Tail }} \leq t \text { or } t_{j} \leq t \mid t_{C I j}>t_{\text {Tail }}\right) \\
& =P\left(r_{j}^{\prime}+t_{\text {Tail }} \leq t \mid t_{C I j}>t_{\text {Tail }}\right)+P\left(t_{j} \leq t \mid t_{C I j}>t_{\text {Tail }}\right) \\
& \quad-P\left(\left(r_{j}^{\prime}+t_{\text {Tail }} \leq t, t_{j} \leq t\right) \mid t_{C I j}>t_{\text {Tail }}\right) \\
& =P\left(r_{j}^{\prime}+t_{\text {Tail }} \leq t\right)+P\left(t_{j} \leq t\right)-P\left(r_{j}^{\prime}+t_{\text {Tail }} \leq t, t_{j} \leq t\right) \\
& =P\left(t_{p} \leq t-t_{\text {Tail }}\right)+P\left(t_{j} \leq t\right)-P\left(t_{p} \leq t-t_{\text {Tail }}\right) P\left(t_{j} \leq t\right)
\end{aligned}
$$

where we have the independence of $t_{C I j}$ and $t_{j}$ and the independence of $t_{j}$ and $r_{j}^{\prime}$ [15], [16]. We also apply $P\left(\left(r_{j}^{\prime}+\right.\right.$ $\left.\left.t_{\text {Tail }}\right) \leq t\right)=P\left(t_{p} \leq t-t_{\text {Tail }}\right)$ from the memoryless property of the exponential distribution. Thus, $r_{j}^{\prime}$ has the same distribution as $t_{p}$. Therefore, we have the same conditional pdf and the LT with (8) and (20), respectively. As a conclusion, we have the following theorem.

Theorem 1: For a PS network with exponential CHT and Poisson new session arrivals, the following three statements are held if and only if the CRT is exponentially distributed.

1) The new session's eCHT is exponentially distributed.

2) The handoff session's eCHT in busy mode is exponentially distributed.

3) The handoff session's eCHT in idle mode with new or no arrival packets is exponentially distributed.

In summary, Theorem 1 reveals the relationship between eCHT and CRT if either eCHT or CRT is exponentially distributed. The results are the first step in the investigation of eCHT and CRT. For convenience and tractability, many traffic analytical works (e.g., [21]-[23]) in the CS network used the assumption that either CHT or CRT is exponentially distributed. Theorem 1 shows that the assumption also works if both eCHT and CRT are exponentially distributed in the PS network. In the following section, we will relax the restriction of the assumption on exponential CRT to find the associated distribution of CRT.

\section{B. Modeling eCHT by Using LT}

In wired telephone traffic, it is reasonable to assume that the CHTs are exponential distributions. However, the assumption 
of exponential eCHTs in the PS cellular network is only held for the case when the CRTs are exponential distributions (based on our results in Section III-A). In the following, we relax the assumption on exponentially distributed CRTs $\left(f_{d}(t)\right)$ and then use the inverse LT to see how close the exponential distribution is to the eCHT distribution.

Recall that we defined $\lambda, \lambda_{h b}, \lambda_{h d a}$, and $\lambda_{h d b}$ as the arrival rate for new sessions, handoff sessions in busy mode, handoff sessions in idle mode with new arrival packet, and handoff sessions in idle mode without arrival packet, respectively. Thus, according to the weighted average of probability, we can show that $t_{p}=t_{p n h}$ with probability $\lambda /\left(\lambda+\lambda_{h b}+\lambda_{h d a}+\lambda_{h d b}\right)$, $t_{p}=t_{p h h_{-} \text {busy }}$ with probability $\lambda_{h b} /\left(\lambda+\lambda_{h b}+\lambda_{h d a}+\lambda_{h d b}\right)$, and so on. We obtain that

$$
\begin{aligned}
f_{p}^{*}(s)= & \frac{\lambda}{\lambda+\lambda_{h b}+\lambda_{h d a}+\lambda_{h d b}} f_{p n h}^{*}(s) \\
& +\frac{\lambda_{h b}}{\lambda+\lambda_{h b}+\lambda_{h d a}+\lambda_{h d b}} f_{p h h-\text { busy }}^{*}(s) \\
& +\frac{\lambda_{h d a}}{\lambda+\lambda_{h b}+\lambda_{h d a}+\lambda_{h d b}} f_{p h h-\text { idle-new }}^{*}(s) \\
& +\frac{\lambda_{h d b}}{\lambda+\lambda_{h b}+\lambda_{h d a}+\lambda_{h d b}} f_{p h h-\text { idle-no }}^{*}(s) .
\end{aligned}
$$

We assume that $\lambda_{h d a}=\lambda_{h b}$ and $\lambda_{h d b}=0$. From (18), we have

$$
\begin{aligned}
f_{p}^{*}(s)= & \frac{\lambda}{2 \lambda+\lambda_{h b}}\left[f_{p h h}^{*}(s)+f_{p h h \_ \text {idle_new }}^{*}(s)\right] \\
& +\frac{\lambda_{h b}}{2 \lambda+\lambda_{h b}} f_{p h h \_ \text {busy }}^{*}(s) .
\end{aligned}
$$

1) Handoff Session in Busy Mode: Applying LT to (9), we obtain

$$
\begin{aligned}
& f_{p h h_{\text {b busy }}}^{*}(s) \\
& =f_{d}^{*}(s)+e^{-t_{\text {Tail }} s} f_{p}^{*}(s)-\int_{0}^{\infty} e^{-s t}\left[f_{d}(t) \int_{0}^{t} f_{p}\left(\tau-t_{\text {Tail }}\right) d \tau\right] d t \\
& \quad-\int_{0}^{\infty} e^{-s t}\left[f_{p}\left(t-t_{\text {Tail }}\right) \int_{0}^{t} f_{d}(\tau) d \tau\right] d t \\
& =f_{d}^{*}(s)+e^{-t_{\text {Tail }} s} f_{p}^{*}(s)-\int_{0}^{\infty} e^{-s t}\left(1-e^{-\mu\left(t-t_{\text {Tail }}\right)}\right) f_{d}(t) d t \\
& \quad-\mu e^{\mu t_{\text {Tail }}} \int_{0}^{\infty} e^{-(s+\mu) t} \int_{0}^{t} f_{d}(\tau) d \tau d t \\
& =f_{d}^{*}(s)+e^{-t_{\text {Tail }} s} f_{p}^{*}(s)-\mu e^{\mu t_{\text {Tail }}} \int_{0}^{\infty} e^{-(s+\mu) t} F_{d}(t) d t \\
& \quad-\left[\int_{0}^{\infty} e^{-s t} f_{d}(t) d t-e^{\mu t_{\text {Tail }}} \int_{0}^{\infty} e^{-(s+\mu) t} f_{d}(t) d t\right] \\
& =f_{d}^{*}(s)+e^{-t_{\text {Tail }} s} f_{p}^{*}(s)-f_{d}^{*}(s)+e^{\mu t_{\text {Tail }}} f_{d}^{*}(s+\mu) \\
& \quad-\mu e^{\mu t_{\text {Tail }}} \frac{1}{s+\mu} f_{d}^{*}(s+\mu) \\
& =e^{-t_{\text {Tail }} s} \frac{\mu}{s+\mu}+e^{\mu t_{\text {Tail }}}\left(\frac{s}{s+\mu}\right) f_{d}^{*}(s+\mu)
\end{aligned}
$$

where we apply the property of LT that $\mathcal{L}\left(g^{\prime}(t)\right)=s \mathcal{L}(g(t))-$ $g(0)$, if $g$ is continuous at $t=0$. Here, $g(t)=F_{d}(t), g^{\prime}(t)=$ $f_{d}(t)$, and $f_{d}(0)=0$.

2) New Session: Thus, the LT of (15) is

$$
\begin{aligned}
& f_{p n h}^{*}(s) \\
& =f_{r}^{*}(s)+e^{-t_{\text {Tail }} s} f_{p}^{*}(s)-\int_{0}^{\infty} e^{-s t}\left[f_{r}(t) \int_{0}^{t} f_{p}\left(\tau-t_{\text {Tail }}\right) d \tau\right] d t \\
& \quad-\int_{0}^{\infty} e^{-s t}\left[f_{p}\left(t-t_{\text {Tail }}\right) \int_{0}^{t} f_{r}(\tau) d \tau\right] d t \\
& =f_{r}^{*}(s)+e^{-t_{\text {Tail }} s} f_{p}^{*}(s)-\int_{0}^{\infty} e^{-s t}\left(1-e^{-\mu\left(t-t_{\text {Tail }}\right)}\right) f_{r}(t) d t \\
& \quad-\mu e^{\mu t_{\text {Tail }}} \int_{0}^{\infty} e^{-(s+\mu) t} \int_{0}^{t} f_{r}(\tau) d \tau d t \\
& =f_{r}^{*}(s)+e^{-t_{\text {Tail }} s} f_{p}^{*}(s)-\mu e^{\mu t_{\text {Tail }}} \int_{0}^{\infty} e^{-(s+\mu) t} F_{r}(t) d t \\
& \quad-\left[\int_{0}^{\infty} e^{-s t} f_{r}(t) d t-e^{\mu t_{\text {Tail }}} \int_{0}^{\infty} e^{-(s+\mu) t} f_{r}(t) d t\right] \\
& =f_{r}^{*}(s)+e^{-t_{\text {Tail }} s} f_{p}^{*}(s)-f_{r}^{*}(s)+e^{\mu t_{\text {Tail }}} f_{r}^{*}(s+\mu) \\
& -\mu e^{\mu t_{\text {Tail }}} \frac{1}{s+\mu} f_{r}^{*}(s+\mu) \\
& =e^{-t_{\text {Tail }} s} \frac{\mu}{s+\mu}+e^{\mu t_{\text {Tail }}}\left(\frac{s}{s+\mu}\right) f_{r}^{*}(s+\mu) .
\end{aligned}
$$

Furthermore, we need to investigate the relationship between $f_{r}^{*}(s)$ and $f_{d}^{*}(d)$. Because the residual lifetime $r_{1}$ can be viewed as the residual life of the CRT, from the Residual Life Theorem [32], [35], we obtain

$$
f_{r}^{*}(s)=\frac{\eta\left[1-f_{d}^{*}(d)\right]}{s}
$$

where $\eta=\left[\sum_{i=1}^{M} \alpha_{i} / \eta_{i}\right]^{-1}$.

If the residual lifetime $r_{1}$ of $t_{1}$ is exponentially distributed with parameter $\mu_{r}$, the $\mathrm{LT}$ of $r_{1}\left(f_{r}^{*}(s)\right)$ is $\mu_{r} /\left(s+\mu_{r}\right)$. Taking this into (21), we obtain

$$
\begin{aligned}
f_{p n h}^{*}(s) & =e^{-t_{\text {Tail }} s} \frac{\mu}{s+\mu}+e^{\mu t_{\text {Tail }}}\left(\frac{s}{s+\mu}\right) f_{r}^{*}(s+\mu) \\
& =e^{-t_{\text {Tail }} s} \frac{\mu}{s+\mu}+e^{\mu t_{\text {Tail }}} \frac{\mu_{r} s}{(s+\mu)\left(s+\mu+\mu_{r}\right)} .
\end{aligned}
$$

Applying simple poles (see Appendix) or looking up (21) in the table of inverse LTs, we can obtain

$f_{p n h}(t)$

$$
\begin{aligned}
& =u_{a}(t) \mu e^{-\mu\left(t-t_{\text {Tail }}\right)}+e^{\mu t_{\text {Tail }}} \frac{\mu_{r}\left(-\mu e^{-\mu t}-\left(-\mu-\mu_{r}\right) e^{-\left(\mu+\mu_{r}\right) t}\right)}{-\mu-\left(-\mu-\mu_{r}\right)} \\
& =u_{a}(t) \mu e^{-\mu\left(t-t_{\text {Tail }}\right)}-e^{\mu t_{\text {Tail }}} \mu e^{-\mu t}+e^{\mu t_{\text {Tail }}}\left(\mu+\mu_{r}\right) e^{-\left(\mu+\mu_{r}\right) t} .
\end{aligned}
$$


Similarly, if $t_{j}$ is exponentially distributed with parameter $\mu_{d}$, we have

$$
\begin{aligned}
& f_{p h h}(t) \\
& =u_{a}(t) \mu e^{-\mu\left(t-t_{\text {Tail }}\right)}+e^{\mu t_{\text {Tail }}} \frac{\mu_{d}\left(-\mu e^{-\mu t}-\left(-\mu-\mu_{d}\right) e^{-\left(\mu+\mu_{d}\right) t}\right)}{-\mu-\left(-\mu-\mu_{d}\right)} \\
& =u_{a}(t) \mu e^{-\mu\left(t-t_{\text {Tail }}\right)}-e^{\mu t_{\text {Tail }}} \mu e^{-\mu t}+e^{\mu t_{\text {Tail }}}\left(\mu+\mu_{d}\right) e^{-\left(\mu+\mu_{d}\right) t} .
\end{aligned}
$$

3) Handoff Session in Idle With New Arrival Packets: Similarly, $t_{p h h_{-} \text {idle_new }}$ in (6) is the same as $t_{p h h_{-} \text {busy }}$ in (5). We have the same conditional pdf and the LT with (8) and (20), respectively.

4) Handoff Session in Idle Without Arrival Packet: Because the conditional pdf of $t_{p h h_{\text {_idle_no }}}$ in (17) is the same as the conditional pdf of $t_{p h h_{-} \text {busy }}$ in (8), the LT of (17) is also the same as that of (20). We conclude our results in Theorem 2 as follows.

Theorem 2: For a PS network with exponential eCHT and Poisson new session arrival rate $\lambda$, we have the following statements.

1) For a new session, the LT of the eCHT's pdf is given by

$$
f_{p n h}^{*}(s)=e^{-t_{\text {Tail }} s} \frac{\mu}{s+\mu}+e^{\mu t_{\text {Tail }}}\left(\frac{s}{s+\mu}\right) f_{r}^{*}(s+\mu) .
$$

2) The LT of the eCHT's pdf for a handoff session in busy mode or a handoff session with new arrival packets or a handoff session without arrival packet in idle mode is given by

$$
\begin{aligned}
f_{p h h_{\_} \text {busy }}^{*}(s) & =f_{p h h_{\_} \text {idle_no }}^{*}(s)=f_{p h h_{\_} \text {idle_new }}^{*}(s) \\
& =e^{-t_{\text {Tail }} s} \frac{\mu}{s+\mu}+e^{\mu t_{\text {Tail }}}\left(\frac{s}{s+\mu}\right) f_{d}^{*}(s+\mu) .
\end{aligned}
$$

3) The LT of the eCHT's pdf is given by

$$
\begin{aligned}
f_{p}^{*}(s)= & \frac{\lambda}{2 \lambda+\lambda_{h b}}\left[f_{p n h}^{*}(s)+f_{p h h \_ \text {idle_new }}^{*}(s)\right] \\
& +\frac{\lambda_{h b}}{2 \lambda+\lambda_{h b}} f_{p h h \_ \text {busy }}^{*}(s) .
\end{aligned}
$$

4) If CRT is exponentially distributed, the eCHT of the new session and the eCHT of the handoff session have the same distribution, and vice versa.

Using Theorem 2, we can obtain the eCHT's pdf by the inverse LT, from which the distribution functions of CRT can be obtained.

\section{Analytical Model of Conditional Distribution \\ With Packet Session's eCHT}

In the CS network, conditional CHT, which is easier to obtain, is proved to have the same distribution as CHT and has been widely used to further reduce computational complexity for the approximation of CHT distribution [10], [15], [36].
Orlik and Rappaport in [10] and [36] derived the results for conditional distributions for the CHT when the CRT is the sum of the hyper-Exponential (SOHYP) distribution. The work in [15] showed that the conditional CHT has the same distribution as the unconditional CHT when CRT is exponentially distributed. The work significantly reduces the CHT's computational complexity and has been widely used (e.g., [37] and [38]). Here, however, we will point out that the results are no longer valid in PS networks.

Let $f_{c n h}(t)$ and $f_{c h h}(t)$ denote the conditional pdf of the new session's eCHT and the conditional pdf of the handoff session's eCHT in busy mode, respectively. Note that the conditional pdf of the handoff session's eCHT in idle mode with (without) new arrival packets $\left(f_{p h h_{-} \text {idle_new }}(t)\right.$ and $\left.f_{p h h_{-} \text {idle_no }}(t)\right)$ is the same as the handoff session's eCHT in busy mode $\left(f_{\text {phh_busy }}(t)\right)$ because (5), (6), and (7) are the same. The corresponding LTs of these pdfs are $f_{c n h}^{*}(t)$ and $f_{c h h}^{*}(t)$, and with cdfs $F_{c n h}(t)$ and $F_{c h h}(t)$, respectively. We first start from $f_{c h h}(t)$ conditioning on the timer, which is a timeout, and $t_{j} \geq r_{j}+t_{\text {Tail }}$. We then obtain the following expressions.

1) Handoff Session (in Busy Mode):

$$
\begin{aligned}
F_{c h h}(h)= & \operatorname{Pr}\left(t_{\text {phh_busy }} \leq h \mid r_{j}+t_{\text {Tail }} \leq t_{j}\right) \\
= & \frac{\operatorname{Pr}\left(r_{j}+t_{\text {Tail }} \leq h, r_{j}+t_{\text {Tail }} \leq t_{j}\right)}{\operatorname{Pr}\left(r_{j}+t_{\text {Tail }} \leq t_{j}\right)} \\
= & \frac{\int_{0}^{h-t_{\text {Tail }}} f_{p}(t) \int_{t+t_{\text {Tail }}}^{\infty} f(\tau) d \tau d t}{\operatorname{Pr}\left(r_{j}+t_{\text {Tail }} \leq t_{j}\right)} \\
= & \frac{\int_{0}^{h-t_{\text {Tail }}} f_{p}(t)\left[1-F_{d}\left(t+t_{\text {Tail }}\right)\right] d t}{\operatorname{Pr}\left(r_{j}+t_{\text {Tail }} \leq t_{j}\right)} .
\end{aligned}
$$

Differentiating both sides, we obtain the following conditional pdf:

$$
f_{\text {chh }}(h)=\frac{f_{p}\left(h-t_{\text {Tail }}\right)\left[1-F_{d}(h)\right]}{\operatorname{Pr}\left(r_{j}+t_{\text {Tail }} \leq t_{j}\right)} .
$$

We observe that

$$
\begin{aligned}
\operatorname{Pr}\left(r_{j}+t_{\text {Tail }} \leq t_{j}\right) & =\int_{t=t_{\text {Tail }}}^{\infty} \int_{0}^{t-t_{\text {Tail }}} f_{d}(t) f_{p}(\tau) d \tau d t \\
& =\int_{t=t_{\text {Tail }}}^{\infty} f_{d}(t)\left[1-e^{-\mu\left(t-t_{\text {Tail }}\right)}\right] d t \\
& =1-\int_{t=t_{\text {Tail }}}^{\infty} f_{d}(t) e^{-\mu\left(t-t_{\text {Tail }}\right)} d t \\
& =1-F_{d}\left(t_{\text {Tail }}\right)-e^{\mu t_{\text {Tail }}} f_{d}^{*}(\mu) .
\end{aligned}
$$

Taking this into (29), we obtain

$$
f_{c h h}(h)=\frac{\left[1-F_{d}(h)\right] \mu e^{-\mu\left(h-t_{\text {Tail }}\right)}}{1-F_{d}\left(t_{\text {Tail }}\right)-e^{\mu t_{\text {Tail }}} f_{d}^{*}(\mu)} .
$$


Hence

$$
\begin{aligned}
f_{c h h}^{*}(s) & =\frac{\mu \int_{0}^{\infty} e^{-(s+\mu)\left(h-t_{\text {Tail }}\right)}\left[1-F_{d}(h)\right] d h}{1-F_{d}\left(t_{\text {Tail }}\right)-e^{\mu t_{\text {Tail }}} f_{d}^{*}(\mu)} \\
= & \frac{\mu e^{(s+\mu) t_{\text {Tail }}}}{s+\mu} \frac{1-f_{d}^{*}(s+\mu)}{1-F_{d}\left(t_{\text {Tail }}\right)-e^{\mu t_{\text {Tail }}} f_{d}^{*}(\mu)} .
\end{aligned}
$$

2) New Session: In a similar fashion, we obtain the following result for the new session's eCHT:

$$
\begin{aligned}
f_{c n h}(h)= & \frac{\left[1-F_{r}(h)\right] \mu e^{-\mu\left(h-t_{\text {Tail }}\right)}}{1-F_{r}\left(t_{\text {Tail }}\right)-e^{\mu t_{\text {Tail }}} f_{r}^{*}(\mu)} \\
f_{c n h}^{*}(s)= & \frac{\mu \int_{0}^{\infty} e^{-(s+\mu)\left(h-t_{\text {Tail }}\right)}\left[1-F_{r}(h)\right] d h}{1-F_{r}\left(t_{\text {Tail }}\right)-e^{\mu t_{\text {Tail }}} f_{r}^{*}(\mu)} \\
= & \frac{\mu e^{(s+\mu) t_{\text {Tail }}}}{s+\mu} \times \frac{1-f_{r}^{*}(s+\mu)}{1-F_{r}\left(t_{\text {Tail }}\right)-e^{\mu t_{\text {Tail }}} f_{r}^{*}(\mu)} .
\end{aligned}
$$

The conditional eCHT distribution $f_{c h}(t)$ is the (weighted) average of the conditional new session and handoff session eCHT distribution. Thus

$$
\begin{aligned}
f_{c h}^{*}(s)= & \frac{\mu e^{(s+\mu) t_{\text {Tail }}}}{s+\mu} \\
& \times\left[\frac{\lambda}{2 \lambda+\lambda_{h}} \cdot \frac{1-f_{r}^{*}(s+\mu)}{1-F_{r}\left(t_{\text {Tail }}\right)-e^{\mu t_{\text {Tail }}} f_{r}^{*}(\mu)}\right. \\
& \left.\quad+\frac{\lambda_{h}}{2 \lambda+\lambda_{h}} \cdot \frac{1-f_{d}^{*}(s+\mu)}{1-F_{d}\left(t_{\text {Tail }}\right)-e^{\mu t_{\text {Tail }}} f_{d}^{*}(\mu)}\right] .
\end{aligned}
$$

Let $T_{c n h}, T_{c h h}$, and $T_{c h}$ denote the expected conditional new session eCHT, the expected conditional handoff session eCHT, and the expected conditional eCHT, respectively. We then have

$$
\begin{aligned}
& T_{c h h}=\frac{-e^{\mu t_{\text {Tail }}}}{\mu} {\left[\frac{1-\mu t_{\text {Tail }}-f^{*}(\mu)}{1-e^{\mu t_{\text {Tail }}} f^{*}(\mu)}+f^{*(1)}(\mu)\right] } \\
& T_{c n h}=\frac{-e^{\mu t_{\text {Tail }}}}{\mu}\left[\frac{1-\mu t_{\text {Tail }}-f_{r}^{*}(\mu)}{1-e^{\mu t_{\text {Tail }}} f_{r}^{*}(\mu)}+f_{r}^{*(1)}(\mu)\right] \\
& T_{c h}=\frac{-e^{\mu t_{\text {Tail }}}}{\mu}\left\{\frac{1-\mu t_{\text {Tail }}-f_{r}^{*}(\mu)}{1-e^{\mu t_{\text {Tail }}} f_{r}^{*}(\mu)}+f_{r}^{*(1)}(\mu)\right. \\
&\left.+\frac{\lambda_{h}}{2 \lambda+\lambda_{h}}\left[\frac{1-\mu t_{\text {Tail }}-f^{*}(\mu)}{1-e^{\mu t_{\text {Tail }}} f^{*}(\mu)}+f^{*(1)}(\mu)\right]\right\} .
\end{aligned}
$$

When the residual lifetime $r_{1}$ of $t_{1}$ is exponentially distributed with parameter $\mu_{r}$, then its LT is $f_{r}^{*}(s)=\mu_{r} /\left(s+\mu_{r}\right)$. From (31), we obtain

$$
\begin{aligned}
f_{c n h}^{*}(s) & =\frac{\mu e^{(s+\mu) t_{\text {Tail }}}}{s+\mu} \cdot \frac{1-f_{r}^{*}(s+\mu)}{1-F_{r}\left(t_{\text {Tail }}\right)-e^{\mu t_{\text {Tail }}} f_{r}^{*}(\mu)} \\
& =\frac{\mu e^{(s+\mu) t_{\text {Tail }}}}{s+\mu} \times \frac{1-\frac{\mu_{r}}{s+\mu+\mu_{r}}}{1-\left(1-\mu e^{\left.-\mu t_{\text {Tail }}\right) e^{\mu t_{\text {Tail }}} \frac{\mu_{r}}{\mu+\mu_{r}}}\right.} \\
& =\frac{\mu e^{(s+\mu) t_{\text {Tail }}}}{s+\mu} \cdot \frac{\frac{s+\mu}{s+\mu+\mu_{r}}}{\frac{\mu \mu_{r}}{\mu+\mu_{r}}} \\
& =\frac{e^{(s+\mu) t_{\text {Tail }}}}{s+\mu+\mu_{r}} \cdot \frac{\mu+\mu_{r}}{\mu_{r}} .
\end{aligned}
$$

\section{Modeling of Handoff Rate for General eCHTs and CRTs}

As pointed out in the introduction, it is necessary to study the handoff rate and its impact on eCHT. Here, we study the handoff rate for the general case where the eCHTs and CRTs are generally distributed. The handoff rate is defined as the average number of handoffs during an ongoing session. This value is used to characterize the eCHT distribution and is related to the service quality or cost parameters.

Let $t_{1}, t_{2}, \ldots$ denote the CRT (they are independent and identically distributed (i.i.d.) nonlattice distributions) and $r_{1}$ denote the residual life of a new session. Let $t_{p}$ denote the CHT for the packet session. Let $H$ be the number of handoffs of a packet session (without blocking) during the session connection.

$H=0$ means the session is not blocked, and the eCHT $t_{p}+$ $t_{\text {Tail }}$ is shorter than the residual life $r_{1}$ (the session completes before the portable moves out of the cell). If $H=1$ means the session is not initially blocked, the UE either makes a successful handoff and then completes the session successfully in the new cell or is forced to terminate because of the first handoff failure, and so on. If the blocking probability for a new session is $p_{0}$ and the probability for a handoff session to be forced to terminate is $p_{f}$, we have

$$
\begin{aligned}
\operatorname{Pr}(H=0)= & \left(1-p_{0}\right) \operatorname{Pr}\left(r_{1} \geq t_{p}+t_{\text {Tail }}\right) \\
\operatorname{Pr}(H=1)= & \left(1-p_{0}\right) \operatorname{Pr}\left(r_{1}<t_{p}+t_{\text {Tail }} \leq r_{1}+t_{2}\right)\left(1-p_{f}\right) \\
& +\left(1-p_{0}\right) \operatorname{Pr}\left(t_{p}+t_{\text {Tail }}>r_{1}+t_{2}\right) p_{f} \\
& \vdots \\
\operatorname{Pr}(H=k)= & B_{1}
\end{aligned}
$$

where $B_{1}=\left(1-p_{0}\right) \operatorname{Pr}\left(r_{1}+t_{2}+\cdots+t_{k}<t_{p}+t_{\text {Tail }} \leq r_{1}+\right.$ $\left.t_{2}+\cdots+t_{k+1}\right)\left(1-p_{f}\right)^{k}+\left(1-p_{0}\right) \operatorname{Pr}\left(t_{p}+t_{\text {Tail }}>r_{1}+t_{2}+\right.$ $\left.\cdots+t_{k}\right)\left(1-p_{f}\right)^{k-1} p_{f}$.

We first calculate $\operatorname{Pr}(H=0)$ as follows:

$$
\begin{aligned}
\operatorname{Pr} & H=0) \\
& =\left(1-p_{0}\right) \int_{0}^{\infty} \operatorname{Pr}\left(r_{1} \geq t+t_{\text {Tail }}\right) f_{p}(t) d t \\
& =\left(1-p_{0}\right) \int_{0}^{\infty} \int_{t+t_{\text {Tail }}}^{\infty} f_{r}(\tau) d \tau f_{p}(t) d t \\
& =\left(1-p_{0}\right) \int_{0}^{\infty} \frac{1}{2 \pi j} \int_{\sigma-j \infty}^{\sigma+j \infty} \frac{1-f_{r}^{*}\left(s-t_{\text {Tail }}\right)}{s-t_{\text {Tail }}} e^{s t} d s f_{p}(t) d t \\
& =\frac{\left(1-p_{0}\right)}{2 \pi j} \int_{\sigma-j \infty}^{\sigma+j \infty} \frac{1-f_{r}^{*}\left(s-t_{\text {Tail }}\right)}{s-t_{\text {Tail }}} \int_{0}^{\infty} f_{p}(t) e^{s t} d t d s \\
& =\frac{\left(1-p_{0}\right)}{2 \pi j} \int_{\sigma-j \infty}^{\sigma+j \infty} \frac{1-f_{r}^{*}\left(s-t_{\text {Tail }}\right)}{s-t_{\text {Tail }}} f_{p}^{*}(-s) d s .
\end{aligned}
$$

For $k>0$, let $\xi=r_{1}+t_{2}+\cdots+t_{k}$, we have

$$
\begin{aligned}
f_{\xi}^{*}(s) & =E\left[e^{-s \xi}\right]=E\left[e^{-s r_{1}}\right] \Pi_{i=2}^{k} E\left[e^{-s t_{i}}\right] \\
& =f_{r}^{*}(s)\left(f^{*}(s)\right)^{k-1} .
\end{aligned}
$$

The pdf is given by

$$
f_{\xi}(t)=\frac{1}{2 \pi j} \int_{\sigma-j \infty}^{\sigma+j \infty} f_{r}^{*}(s)\left(f^{*}(s)\right)^{k-1} e^{s t} d s
$$


so $L\left(\operatorname{Pr}\left(\xi \leq t+t_{\text {Tail }}\right)\right)=\left(f_{\xi}^{*}\left(s-t_{\text {Tail }}\right) / s-t_{\text {Tail }}\right)$. Thus, we have

$$
\begin{aligned}
\operatorname{Pr} & \left.r_{1}+t_{2}+\cdots+t_{k} \leq t_{p}+t_{\text {Tail }}\right) \\
= & \int_{0}^{\infty} \operatorname{Pr}\left(\xi \leq t+t_{\text {Tail }}\right) f_{p}(t) d t \\
= & \int_{0}^{\infty} \frac{1}{2 \pi j} \int_{\sigma-j \infty}^{\sigma+j \infty} \frac{f_{r}^{*}\left(s-t_{\text {Tail }}\right)\left[f^{*}\left(s-t_{\text {Tail }}\right)\right]^{k-1}}{s-t_{\text {Tail }}} e^{s t} d s f_{p}(t) d t \\
= & \frac{1}{2 \pi j} \int_{\sigma-j \infty}^{\sigma+j \infty} \times \frac{f_{r}^{*}\left(s-t_{\text {Tail }}\right)\left[f^{*}\left(s-t_{\text {Tail }}\right)\right]^{k-1}}{s-t_{\text {Tail }}} f_{p}^{*}(-s) d s .
\end{aligned}
$$

We can obtain

$$
\begin{aligned}
& \operatorname{Pr}(H=k) \\
& =\left(1-p_{0}\right)\left[\operatorname{Pr}\left(t_{p}+t_{\text {Tail }} \geq r_{1}+t_{2}+\cdots+t_{k}\right)\right. \\
& \quad-\operatorname{Pr}\left(t_{p}+t_{\text {Tail }} \geq r_{1}+t_{2}+\cdots+t_{k+1}\right]\left(1-p_{f}\right)^{k} \\
& \quad+\left(1-p_{0}\right) \operatorname{Pr}\left(t_{p}+t_{\text {Tail }} \geq r_{1}+t_{2}+\cdots+t_{k}\right)\left(1-p_{f}\right)^{k-1} p_{f} \\
& =\frac{\left(1-p_{0}\right)\left(1-p_{f}\right)^{k-1}}{2 \pi j} \times \int_{\sigma-j \infty}^{\sigma+j \infty} \frac{f_{r}^{*}\left(s-t_{\text {Tail }}\right)\left[f^{*}\left(s-t_{\text {Tail }}\right)\right]^{k-1}}{s-t_{\text {Tail }}} \\
& \quad \times\left[1-\left(1-p_{f}\right) f^{*}\left(s-t_{\text {Tail }}\right)\right] f_{p}^{*}(-s) d s .
\end{aligned}
$$

Now, we find the Z-transform for the number of handoffs. Let $H(z)$ be the moment-generating function, then we have

$$
\begin{aligned}
& H[z]=E\left[z^{H}\right]=\sum_{k=0}^{\infty} z^{k} \operatorname{Pr}(H=k) \\
& =\operatorname{Pr}(H=0)+\sum_{k=1}^{\infty} z^{k} \operatorname{Pr}(H=k) \\
& =\operatorname{Pr}(H=0)+\frac{\left(1-p_{0}\right) z}{2 \pi j} \\
& \times \int_{\sigma-j \infty}^{\sigma+j \infty} \frac{f_{r}^{*}\left(s-t_{\text {Tail }}\right)\left[1-\left(1-p_{f}\right) f^{*}\left(s-t_{\text {Tail }}\right)\right]}{s-t_{\text {Tail }}} \\
& \times\left\{\sum_{k=1}^{\infty}\left[\left(1-p_{f}\right) z f^{*}\left(s-t_{\text {Tail }}\right)\right]^{k-1}\right\} f_{p}^{*}(-s) d s \\
& =\frac{1-p_{0}}{2 \pi j} \cdot \int_{\sigma-j \infty}^{\sigma+j \infty} \frac{A_{1} \times f_{p}^{*}(-s)}{\left(s-t_{\text {Tail }}\right)\left[1-\left(1-p_{f}\right) z f^{*}\left(s-t_{\text {Tail }}\right)\right]} d s \\
& =\frac{1-p_{0}}{2 \pi j} \cdot \int_{\sigma-j \infty}^{\sigma+j \infty} \frac{A_{2} \times f_{p}^{*}(-s)}{\left(s-t_{\text {Tail }}\right)^{2}\left[1-\left(1-p_{f}\right) z f^{*}\left(s-t_{\text {Tail }}\right)\right]} d s
\end{aligned}
$$

where $A_{1}=1+\left(z-1+z\left(1-\left(1-p_{f}\right) f^{*}\left(s-t_{\text {Tail }}\right)\right)\right) f_{r}^{*}(s-$ $\left.t_{\text {Tail }}\right)-z\left(1-p_{f}\right) f^{*}\left(s-t_{\text {Tail }}\right)$, and $A_{2}=\left(s-t_{\text {Tail }}\right)+(2 z-1-$ $\left.z\left(1-p_{f}\right) f^{*}\left(s-t_{\text {Tail }}\right)\right) \eta\left(1-f^{*}\left(s-t_{\text {Tail }}\right)\right)-z\left(s-t_{\text {Tail }}\right)(1-$ $\left.p_{f}\right) f^{*}\left(s-t_{\text {Tail }}\right)$.

If we use $\sigma_{p}$ to denote the singular points of $f_{p}^{*}(-s)$ in the right half complex plane, from Cauchy's residue theorem, we obtain

$$
\begin{aligned}
H(z)= & -\left(1-p_{0}\right) \\
& \times \sum_{p \in \sigma_{p}} \operatorname{Res}_{s=p} \frac{A_{3} \times f_{p}^{*}(-s)}{\left(s-t_{\text {Tail }}\right)^{2}\left[1-\left(1-p_{f}\right) z f^{*}\left(s-t_{\text {Tail }}\right)\right]}
\end{aligned}
$$

where $A_{3}=\left(s-t_{\text {Tail }}\right)+\left(2 z-1-z\left(1-p_{f}\right) f^{*}\left(s-t_{\text {Tail }}\right)\right) \eta(1-$ $\left.f^{*}\left(s-t_{\text {Tail }}\right)\right)-z\left(s-t_{\text {Tail }}\right)\left(1-p_{f}\right) f^{*}\left(s-t_{\text {Tail }}\right)$, and $\operatorname{Res}_{p}$ denotes the residue at singular point $s=p$. Differentiating $H(z)$ in (36) at $z=1$, we obtain

$$
\begin{aligned}
& E[H]=\frac{1-p_{0}}{2 \pi j} \\
& \times \int_{\sigma-j \infty}^{\sigma+j \infty} \frac{A_{4}}{\left(s-t_{\text {Tail }}\right)^{2}\left[1-\left(1-p_{f}\right) f^{*}\left(s-t_{\text {Tail }}\right)\right]} f_{p}^{*}(-s) d s
\end{aligned}
$$

where $A_{4}=\left(s-t_{\text {Tail }}\right)+\left(1-\left(1-p_{f}\right) f^{*}\left(s-t_{\text {Tail }}\right)\right) \eta(1-$ $\left.f^{*}\left(s-t_{\text {Tail }}\right)\right)-\left(s-t_{\text {Tail }}\right)\left(1-p_{f}\right) f^{*}\left(s-t_{\text {Tail }}\right)$.

Applying Cauchy's residue theorem, we obtain

$E[H]=-\left(1-p_{0}\right)$

$$
\times \sum_{p \in \sigma_{p}} \operatorname{Res}_{s=p} \frac{A_{4} \times f_{p}^{*}(-s)}{\left(s-t_{\text {Tail }}\right)^{2}\left[1-\left(1-p_{f}\right) f^{*}\left(s-t_{\text {Tail }}\right)\right]} .
$$

If the eCHTs are exponentially distributed with parameter $\mu$, $f_{p}^{*}(-s)=\mu /(-s+\mu)$, which has a singular point, and $\sigma_{p}=$ $\{\mu\}$, we obtain

$$
E[H]=\mu\left(1-p_{0}\right) \frac{A_{5}}{\left(\mu-t_{\text {Tail }}\right)^{2}\left[1-\left(1-p_{f}\right) f^{*}\left(\mu-t_{\text {Tail }}\right)\right]}
$$

where $A_{5}=\left(\mu-t_{\text {Tail }}\right)-\left(\mu-t_{\text {Tail }}\right)\left(1-p_{f}\right) f^{*}\left(\mu-t_{\text {Tail }}\right)+$ $\left(1-\left(1-p_{f}\right) f^{*}\left(\mu-t_{\text {Tail }}\right)\right) \eta\left(1-f^{*}\left(\mu-t_{\text {Tail }}\right)\right)$.

If there is no blocking and no forced termination, $p_{0}=p_{f}=0$, and from (39), $E[H]=1$. If there are handoff failures, the expected number of handoffs is intuitively smaller.

Let us assume that the eCHTs are i.i.d. with an Erlang distribution, i.e.,

$$
f_{p}(t)=\frac{\alpha^{m} t^{m-1}}{(m-1) !} e^{-\alpha t}, f_{p}^{*}(s)=\left(\frac{\alpha}{s+\alpha}\right)^{m}
$$

where $\alpha=m \eta$ is the scale parameter, and $m$ is a positive integer. When $m=1$, it gives the exponential distribution. We can also find a simple formula for the handoff rate. Let

$$
g(s)=\frac{A_{4}}{\left(s-t_{\text {Tail }}\right)^{2}\left[1-\left(1-p_{f}\right) f^{*}\left(s-t_{\text {Tail }}\right)\right]} .
$$


Then, $f_{p}^{*}(-s)=[\alpha /(-s+\alpha)]^{m}$ has a unique singular point $\sigma_{p}=\{\alpha\}$. From (38), we obtain

$$
E[H]=(-1)^{m+1}\left(1-p_{0}\right) \alpha^{m} \frac{g^{(m-1)}(\alpha)}{(m-1) !}, \alpha=m \mu .
$$

For this case, if there is no handoff failure, $\left(p_{0}=p_{f}=\right.$ $0)$, from (41), we have $g(s)=\left(s-t_{\text {Tail }}-\eta\right) /\left(s-t_{\text {Tail }}\right)^{2}$, and $g^{(m-1)}(\alpha)=(-1)^{m-1}\left((m-1) !\left(\alpha-t_{\text {Tail }}\right)+\eta(m !)\right) /(\alpha-$ $\left.t_{\text {Tail }}\right)^{(m+1)}$. From (42), we have

$$
E[H]=\frac{(-1)^{2 m} \alpha^{m}\left(\alpha-t_{\text {Tail }}+m \eta\right)}{\left(\alpha-t_{\text {Tail }}\right)^{m+1}} .
$$

In fact, it can be shown that for the ideal case $\left(p_{0}=p_{f}=0\right)$, the expected number of handoffs for any CRT distribution and any eCHT distribution is $E[H]=\left(\alpha^{m}\left(\alpha-t_{\text {Tail }}+m \eta\right) /(\alpha-\right.$ $\left.\left.t_{\text {Tail }}\right)^{m+1}\right)$. Indeed, from (37), if $p_{f}=0$, we obtain

$$
\begin{aligned}
E[H] & =\frac{1-p_{0}}{2 \pi j} \int_{\sigma-j \infty}^{\sigma+j \infty} \frac{1}{s} f_{p}^{*}(-s) d s \\
& =\left(1-p_{0}\right) \int_{0}^{\infty} f_{p}(t)\left[\frac{1}{2 \pi j} \int_{\sigma-j \infty}^{\sigma+j \infty} \frac{1}{s} f_{p}^{*}(-s) d s\right] d t \\
& =\left(1-p_{0}\right) \int_{0}^{\infty} t f_{p}(t) d t=\left(1-p_{0}\right) \frac{1}{\mu} .
\end{aligned}
$$

Next, we show how to find the handoff session arrival rate from the handoff rate. It is easy to observe that for each nonblocking new session, there will be on average $E[H]$ number of handoff sessions induced. Therefore, the handoff session traffic will have arrival rate $\lambda_{h}=\lambda E[H]$. From (38), we obtain

$$
\begin{aligned}
& \lambda_{h}=-\lambda\left(1-p_{0}\right) \\
& \times \sum_{p \in \sigma_{p}} \operatorname{Res}_{s=p} \frac{A_{4}}{\left(s-t_{\text {Tail }}\right)^{2}\left[1-\left(1-p_{f}\right) f^{*}\left(s-t_{\text {Tail }}\right)\right]} f_{p}^{*}(-s) .
\end{aligned}
$$

If the eCHTs are Erlang distributed according to (40), we have

$$
\lambda_{h}=-\lambda(-1)^{m+1}\left(1-p_{0}\right) \alpha^{m} \frac{g^{(m-1)}(\alpha)}{(m-1) !}, \alpha=m \mu .
$$

The cell traffic intensity can be computed from this formula and the expected CHT.

When the eCHTs are Erlang distributed, we have to compute the derivatives of $g(s)$ for the computation of handoff rate and handoff session arrival rate. However, the explicit expressions for the derivatives of $g(s)$ may be difficult. We therefore develop the following recursive algorithm for their computations. Let

$$
h(s)=\left(s-t_{\text {Tail }}\right)^{2}\left[1-\left(1-p_{f}\right) f^{*}\left(s-t_{\text {Tail }}\right)\right] .
$$

We obtain

$$
\begin{aligned}
h^{(0)}(\alpha)= & \left(\alpha-t_{\text {Tail }}\right)^{2}\left[1-\left(1-p_{f}\right) f^{*}\left(\alpha-t_{\text {Tail }}\right)\right] \\
h^{(1)}(\alpha)= & 2\left(\alpha-t_{\text {Tail }}\right)\left(1-\left(1-p_{f}\right) f^{*}\left(\alpha-t_{\text {Tail }}\right)\right) \\
& -\left(\alpha-t_{\text {Tail }}\right)^{2}\left(\left(1-p_{f}\right) f^{*(1)}\left(\alpha-t_{\text {Tail }}\right)\right) \\
h^{(2)}(\alpha)=2 & \left(1-\left(1-p_{f}\right) f^{*}\left(\alpha-t_{\text {Tail }}\right)\right) \\
& -4\left(\alpha-t_{\text {Tail }}\right)\left(1-p_{f}\right) f^{*(1)}\left(\alpha-t_{\text {Tail }}\right) \\
& -\left(\alpha-t_{\text {Tail }}\right)^{2}\left(1-p_{f}\right) f^{*(2)}\left(\alpha-t_{\text {Tail }}\right) \\
h^{(3)}(\alpha)= & -6\left(1-p_{f}\right) f^{*(1)}\left(\alpha-t_{\text {Tail }}\right) \\
& -6\left(\alpha-t_{\text {Tail }}\right)\left(1-p_{f}\right) f^{*(2)}\left(\alpha-t_{\text {Tail }}\right) \\
& -\left(\alpha-t_{\text {Tail }}\right)^{2}\left(1-p_{f}\right) f^{*(3)}\left(\alpha-t_{\text {Tail }}\right) \\
& -12\left(1-p_{f}\right) f^{*(2)}\left(\alpha-t_{\text {Tail }}\right) \\
& -8\left(\alpha-t_{\text {Tail }}\right)\left(1-p_{f}\right) f^{*(3)}\left(\alpha-t_{\text {Tail }}\right) \\
& -\left(\alpha-t_{\text {Tail }}\right)^{2}\left(1-p_{f}\right) f^{*(4)}\left(\alpha-t_{\text {Tail }}\right) .
\end{aligned}
$$

Since $g(s) h(s)=\left(s-t_{\text {Tail }}\right)+\left(1-\left(1-p_{f}\right) f^{*}\left(s-t_{\text {Tail }}\right)\right) \eta(1-$ $\left.f^{*}\left(s-t_{\text {Tail }}\right)\right)-\left(s-t_{\text {Tail }}\right)\left(1-p_{f}\right) f^{*}\left(s-t_{\text {Tail }}\right)$, differentiating both sides, we obtain $(p>0)$

$$
\begin{aligned}
& \sum_{0}^{p} C_{i}^{p} g^{(i)}(s) h^{(p-i)}(s) \\
& =\left\{\left[1-\left(1-p_{f}\right) f^{*}\left(s-t_{\text {Tail }}\right)\right]\right. \\
& \left.\quad \times\left[s-t_{\text {Tail }}+\eta\left(1-f^{*}\left(s-t_{\text {Tail }}\right)\right)\right]\right\}^{(p)} .
\end{aligned}
$$

From this, we obtain the following recursive algorithm to compute $g^{(m-1)}(\alpha)$ :

$$
\begin{aligned}
g^{(0)}(\alpha)= & \frac{\left(1-\left(1-p_{f}\right) f^{*}\left(\alpha-t_{\text {Tail }}\right)\right)}{h(\alpha)} \\
& \times\left(\alpha-t_{\text {Tail }}+\eta\left(1-f^{*}\left(\alpha-t_{\text {Tail }}\right)\right)\right) \\
g^{(p)}(\alpha)= & -\frac{A_{5}}{h(\alpha)}, p>0
\end{aligned}
$$

where $A_{5}=\sum_{0}^{p-1} C_{i}^{p} g^{(i)}(\alpha) h^{(p-i)}(\alpha)-\left[\left(1-\left(1-p_{f}\right) f^{*}(\alpha-\right.\right.$ $\left.\left.\left.t_{\text {Tail }}\right)\right)\left(\alpha-t_{\text {Tail }}+\eta\left(1-f^{*}\left(\alpha-t_{\text {Tail }}\right)\right)\right)\right]^{(p)}$.

\section{Numerical Results}

Here, we present our numerical results on how the CRT distribution affects the eCHT distribution and handoff rate. For the sake of easy calculation, general applicability, and universal approximation property [32], we use the hyper-Erlang distribution model [11], [15], [19] to analyze the eCHT. Another reason is that some pdf functions may not exist as closed-form LT, e.g., lognormal distribution. 


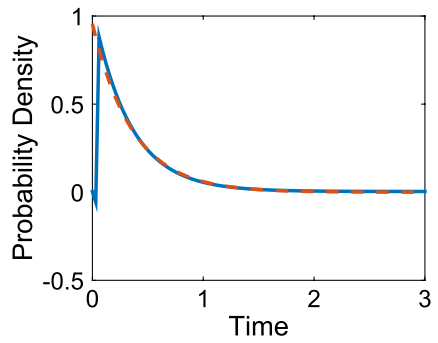

(a)

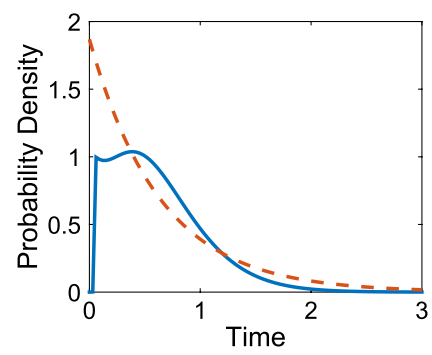

(c)

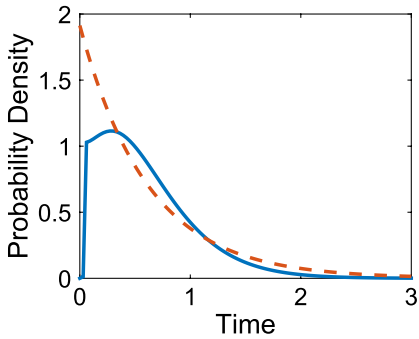

(b)

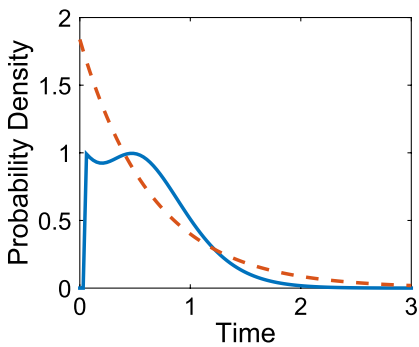

(d)
Fig. 4. PDF of handoff session eCHT (solid line) and its exponential fitting (dashed line) when CRT is Erlang distributed with parameters $(\mu, \eta)$. (a) $m=2$. (b) $m=3$. (c) $m=4$. (d) $m=5$.

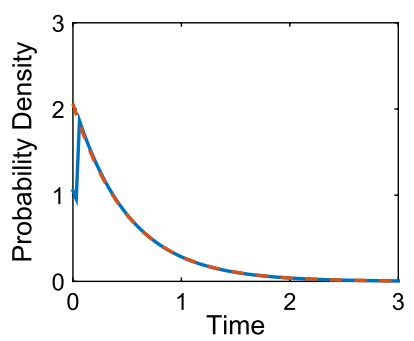

(a)

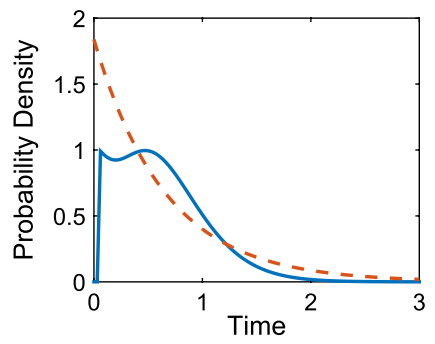

(c)

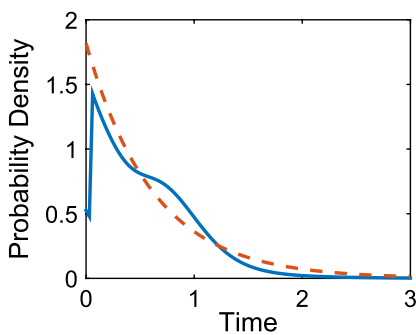

(b)

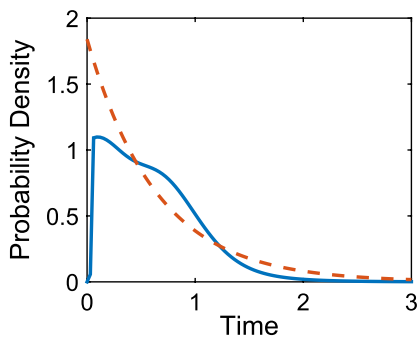

(d)
Fig. 5. PDF of handoff session eCHT (solid line) and its exponential fitting (dashed line) when CRT is hyper-Erlang distributed with two parameters ( $m_{1}, m_{2}, \eta$ ). (a) $m_{1}=1, m_{2}=1$. (b) $m_{1}=1, m_{2}=10$. (c) $m_{1}=5$, $m_{2}=5$. (d) $m_{1}=2, m_{2}=10$.

\section{A. Numerical Results for Section III-B}

We take the Erlang, hyper-Erlang, and exponential (shown in Fig. 4 with $m=1$ ) CRT into Theorem 2. We then can obtain the pdf of the handoff session eCHT from our analytical results.

In Figs. 4 and 5, we show the handoff session's eCHT when CRT is Erlang and hyper-Erlang distributed, respectively. We vary the shape parameter ( $m$ or $m_{1}, m_{2}$ ). It can be seen that the exponential model does not have a good fit for the real handoff session's eCHT distribution when the shape parameter increases.

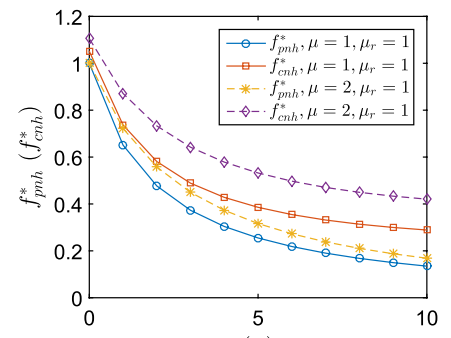

(a)

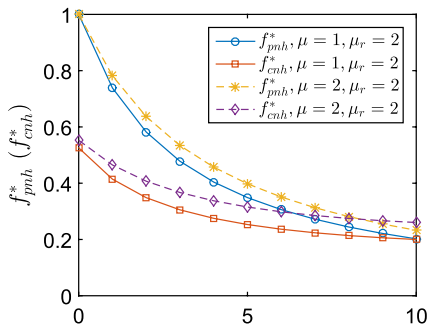

(b)
Fig. 6. Comparison of the LT functions of unconditional $\left(f_{p n h}^{*}\right)$ and conditional $\left(f_{c n h}^{*}\right)$ pdfs of eCHT on $(0,10)$. (a) $s$. (b) $s$.

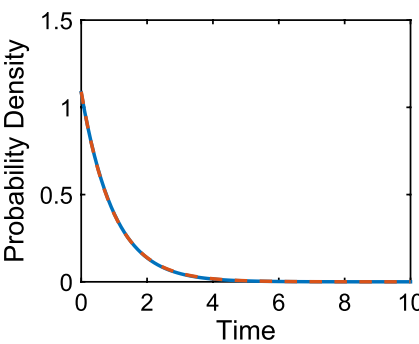

(a)

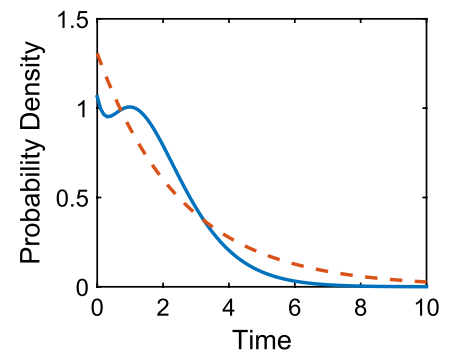

(c)

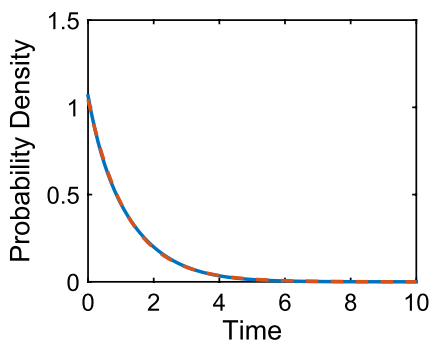

(b)

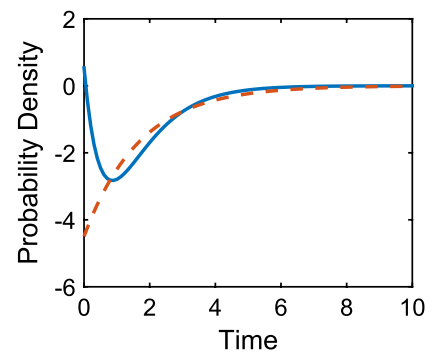

(d)
Fig. 7. Conditional pdf of handoff session eCHT (solid line) and its exponential fitting (dashed line) when CRT is Erlang distributed with parameters $(m, \eta)$. (a) $m=2$. (b) $m=3$. (c) $m=4$. (d) $m=5$.

\section{B. Numerical Results for Section III-C}

Fig. 6 shows the results of the LT functions of unconditional $\left(f_{p n h}^{*}\right)$ and conditional $\left(f_{c n h}^{*}\right)$ pdfs of eCHT, respectively. It shows that the conditional pdf of eCHT cannot be used for the approximation of eCHT as CHT does in the CS network. The finding may prevent researchers from making an inappropriate approximation for eCHT by using its conditional pdf as they usually do in the CS network.

Figs. 7 and 8 show the conditional pdf for the handoff session's eCHT when the CRT is Erlang and hyper-Erlang distributed. From this example, we observe that the conditional distribution for the handoff session's eCHT is a better match to the exponential distribution when the variance is large. However, when the variance becomes small, i.e., the CRT is less spread, this match disappears.

\section{Numerical Results for Section III-D}

Finally, we turn our attention to the handoff rate. Assume that the eCHTs are hyper-Erlang distributed with one parameter. We shall use (42) and the recursive algorithms given at the end of 


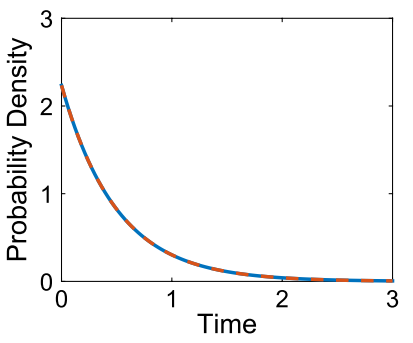

(a)

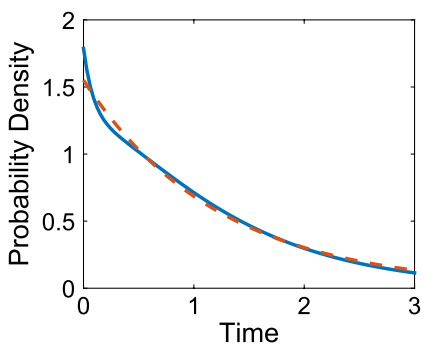

(c)

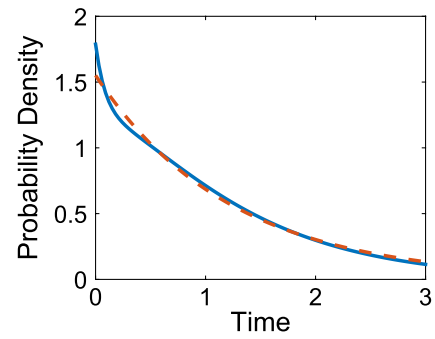

(b)

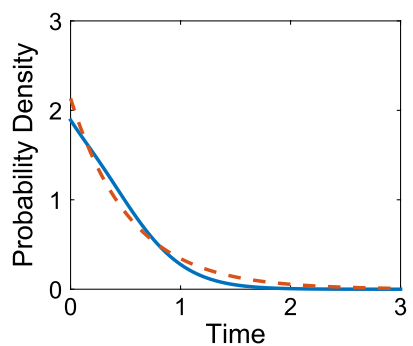

(d)
Fig. 8. Conditional pdf of handoff session eCHT (solid line) and its exponential fitting (dashed line) when CRT is hyper-Erlang distributed with two parameters $\left(m_{1}, m_{2}, \eta\right)$. (a) $m_{1}=1, m_{2}=1$. (b) $m_{1}=1, m_{2}=10$. (c) $m_{1}=5, m_{2}=5$. (d) $m_{1}=2, m_{2}=10$.

the previous section. The CRTs are Gamma distributed with the pdf, i.e.,

$$
f(t)=\frac{\beta^{\gamma} t^{\gamma-1}}{\Gamma(\gamma)} e^{-\beta t}, f^{*}(s)=\left(\frac{\beta}{s+\beta}\right)^{\gamma}, \beta=\gamma \eta
$$

where $\gamma$ is the shape parameter, $\beta$ is the scale parameter, and $\Gamma(\gamma)$ is the Gamma function. The mean and variance of this distribution are $1 / \eta$ and $1 /\left(\gamma \eta^{2}\right)$, respectively.

Figs. 9 and 10 show the handoff rate for different blocking probabilities and forced termination probabilities. We have the following observations.

- The handoff rate for fixed variance of the CRTs (i.e., fixed $m$ ) is increasing when mobility $\eta / \mu$ increases.

- The handoff rate is decreasing for fixed mobility when the variance of the CRTs decreases (i.e., $m$ is increasing).

- The handoff rate is smaller than that in the ideal case (when there is no blocking and no forced termination).

- The handoff rate is insensitive to the variance of the CRTs when mobility $\eta / \mu$ is small, whereas it is very sensitive to the variance of the CRT when mobility $\eta / \mu$ is high.

Since the handoff session arrival rate is equal to the new session arrival rate times the handoff rate in a session, we observe from Figs. 9 and 10 that for highly moving users the handoff rate is much smaller for the hyper-Erlang distributed with one parameter eCHT $(m>1)$ than that for exponential eCHT $(m=1)$. This implies that the exponential assumption for eCHT overestimates the handoff rate. This suggests that PS network designers have to carefully consider the distribution model for eCHT and CRT to meet the blocking probability requirement: Exponential distribution approximation for CRTs and eCHTs may not be enough for the real PS network design.

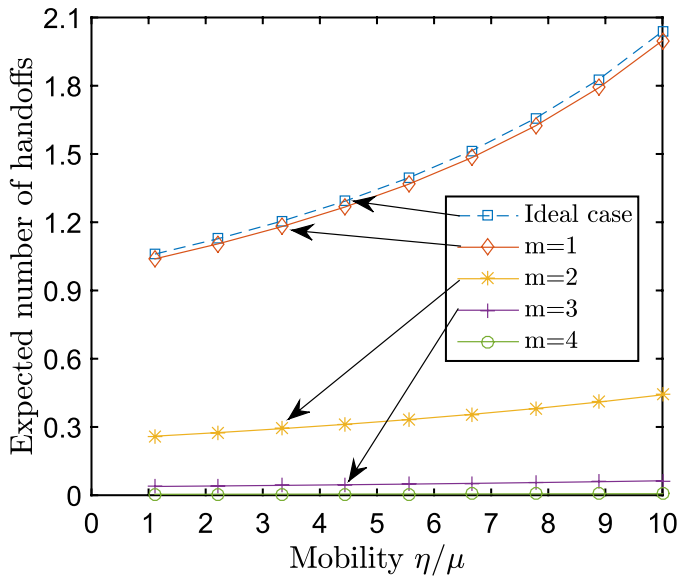

Fig. 9. Handoff rate: dashed line for the ideal case $\left(p_{0}=p_{f}=0\right)$ and solid lines for $m=1 \sim 4$ with $p_{f}=p_{0}=0.02$.

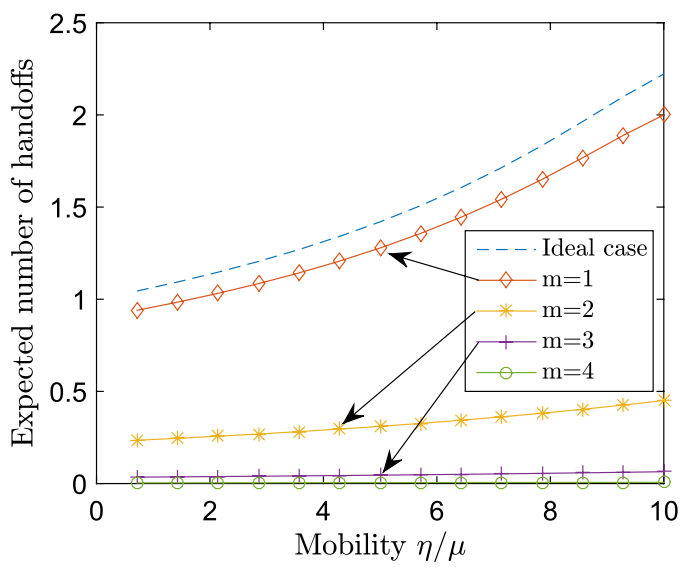

Fig. 10. Handoff rate: dashed line for the ideal case $\left(p_{0}=p_{f}=0\right)$ and solid lines for $m=1 \sim 4$ with $p_{f}=0.05$ and $p_{0}=0.01$.

\section{CONCLusion}

In this paper, we have proposed new models with DRX mode to analyze the impacts of eCHT for packet sessions in PS cellular networks. The new models reveal the essential relationship between eCHT, CRT, and handoff rate for traffic modeling and performance analysis in PS networks. Our analytical results show that some characteristics of eCHT are similar with those of CHT in CS networks so that researchers can use the same assumptions and results derived in the CS network for their performance modeling. However, we also point out the inapplicable assumptions and results of CHT in PS networks. The results can prevent researchers from making inappropriate assumptions on performance modeling and are essential for the traffic study, management, and performance evaluation of PS cellular networks. The results also enable wide applicability in various scenarios and therefore have important theoretical significance. The models presented in this paper can be used as a theoretical guideline for network designers and performance analysts. Our future work would be to study the models for configuring the appropriate parameters for mobile cellular networks. 


\section{APPENDIX} for

Simple poles [39]: Suppose that we have LT $F(S)=\mathcal{L}(f(t))$

$$
\begin{aligned}
F(s) & =\frac{P(s)}{Q(s)} \\
& =\frac{P(s)}{\left(s-\alpha_{1}\right)\left(s-\alpha_{2}\right) \cdots\left(s-\alpha_{n}\right)}, \alpha_{i} \neq \alpha_{j}
\end{aligned}
$$

where $P(s)$ is a polynomial of degree less than $n$. The $\alpha_{i}$ 's are known as simple poles of $F(s)$ in the terminology of complex variables. Partial-fraction decomposition is

$$
F(s)=\frac{A_{1}}{\left(s-\alpha_{1}\right)}+\frac{A_{2}}{\left(s-\alpha_{2}\right)}+\cdots+\frac{A_{n}}{\left(s-\alpha_{n}\right)} .
$$

By multiplying both sides of (48) by $s-\alpha_{i}$ and letting $s \rightarrow$ $\alpha_{i}$, we obtain

$$
A_{i}=\lim _{s \rightarrow \alpha_{i}}\left(s-\alpha_{i}\right) F(s) .
$$

Thus

$$
\begin{aligned}
f(t) & =\mathcal{L}^{-1}(F(s)) \\
& =\sum_{i=1}^{n} \mathcal{L}^{-1}\left(\frac{A_{i}}{s-\alpha_{i}}\right)=\sum_{i=1}^{n} A_{i} e^{\alpha_{i} t} .
\end{aligned}
$$

According to (49), $A_{i}$ gives a quick method, i.e.,

$$
f(t)=\mathcal{L}^{-1}(F(s))=\sum_{i=1}^{n} \lim _{s \rightarrow \alpha_{i}}\left(s-\alpha_{i}\right) F(s) e^{\alpha_{i} t} .
$$

After applying it to (22), we can find

$$
\begin{aligned}
\mathcal{L}^{-1} & {\left[\frac{\mu_{r} s}{(s+\mu)\left(s+\mu+\mu_{r}\right)}\right] } \\
= & \mathcal{L}^{-1}\left[\frac{\mu_{r} s}{(s-(-\mu))\left(s-\left(\mu-\mu_{r}\right)\right)}\right] .
\end{aligned}
$$

From (50), therefore, we can get

$$
\begin{aligned}
f(t)= & \mathcal{L}^{-1}(F(s)) \\
= & \lim _{s \rightarrow-\mu}(s+\mu) F(s) e^{-\mu t} \\
& +\lim _{s \rightarrow-\mu-\mu_{r}}\left(s+\mu+\mu_{r}\right) F(s) e^{-\left(\mu+\mu_{r}\right) t} \\
= & \frac{-\mu \cdot \mu_{r}}{-\mu+\mu+\mu_{r}} \cdot e^{-\mu t}+\frac{\mu_{r} \cdot\left(-\mu-\mu_{r}\right)}{\left(-\mu-\mu_{r}\right)+\mu} \cdot e^{-\left(\mu+\mu_{r}\right) t} .
\end{aligned}
$$

\section{REFERENCES}

[1] "Ericsson mobility report: On the pulse of the networked society," Ericsson, Stockholm, Sweden, Nov. 2015.

[2] Y. B. Lin and A. C. Pang, Wireless and Mobile All-IP Networks. Chichester, U.K.: Wiley, 2005.

[3] S. Jeon, N. Kang, D. Corujo, and R. L. Aguiar, "Comprehensive performance evaluation of distributed and dynamic mobility routing strategy," Elsevier Comput. Netw., vol. 79, pp. 53-67, Jan. 2015.

[4] M. Ismail, A. Abdrabou, and W. Zhuang, "Cooperative decentralized resource allocation in heterogeneous wireless access medium," IEEE Trans. Wireless Commun., vol. 12, no. 2, pp. 714-724, Feb. 2013.

[5] S.-I. Sou, "Mobile data offloading with policy and charging control in 3GPP core network," IEEE Trans. Veh. Technol., vol. 62, no. 7, pp. 3481-3486, Sep. 2013.

[6] B. Zhao, Y. Chen, C. He, and L. Jiang, "Performance analysis of spectrum sensing with multiple primary users," IEEE Trans. Veh. Technol., vol. 61, no. 2, pp. 914-918, Feb. 2012.
[7] T.-C. Chen, J.-C. Chen, and Z.-H. Liu, "Secure network mobility (SeNEMO) for real-time applications," IEEE Trans. Mobile Comput., vol. 10, no. 8, pp. 1113-1130, Aug. 2011.

[8] T. Guo, A. ul Quddus, and R. Tafazolli, "Seamless handover for LTE macro-femto networks based on reactive data bicasting," IEEE Commun. Lett., vol. 16, no. 11, pp. 1788-1791, Nov. 2012.

[9] D. Hong and S. S. Rappaport, "Traffic model and performance analysis for cellular mobile radio telephone systems with prioritized and nonprioritized handoff procedures," IEEE Trans. Veh. Technol., vol. 35, no. 3, pp. 77-92, Aug. 1986.

[10] P. V. Orlik and S. S. Rappaport, "A model for teletraffic performance and channel holding time characterization in wireless cellular communication with general session and dwell time distributions," IEEE J. Sel. Areas Commun., vol. 16, no. 5, pp. 788-803, Jun. 1998.

[11] Y. Fang, I. Chlamtac, and Y. B. Lin, "Channel occupancy times and handoff rate for mobile computing and PCS networks," IEEE Trans. Comput., vol. 47, no. 6, pp. 679-692, Jun. 1998.

[12] E. D. Re, R. Fantacci, and G. Giambene, "Handover and dynamic channel allocation techniques in mobile cellular networks," IEEE Trans. Veh. Technol., vol. 44, no. 2, pp. 229-237, May 1995.

[13] A. L. E. Corral-Ruiz, A. Rico-Páez, F. A. Cruz-Pérez, and G. Hernández-Valdez, "On the functional relationship between channel holding time and cell dwell time in mobile cellular networks," in Proc. IEEE GLOBECOM, 2010, pp. 1-6.

[14] C. Jedrzycki and V. Leung, "Probability distributions of channel holding time in cellular telephony systems," in Proc. IEEE VTC, Apr. 1996, pp. 247-251.

[15] Y. Fang and I. Chlamtac, "Teletraffic analysis and mobility modeling of PCS networks," IEEE Trans. Commun., vol. 47, no. 7, pp. 1062-1072, Jul. 1999.

[16] M. M. Zonoozi and P. Dassanayake, "User mobility modeling and characterization of mobility patterns," IEEE J. Sel. Areas Commun., vol. 15, no. 7, pp. 1239-1252, Sep. 1997.

[17] X. Wang and P. Fan, "Channel holding time in wireless cellular communications with general distributed session time and dwell time," IEEE Commun. Lett., vol. 11, no. 2, pp. 158-160, Feb. 2007.

[18] S. Pattaramalai, V. Aalo, and G. Efthymoglou, "Call completion probability with Weibull distributed call holding time and cell dwell time," in Proc. IEEE GLOBECOM, Nov. 2007, pp. 2634-2638.

[19] Y. Fang, "Hyper-Erlang distribution model and its application in wireless mobille networks," Wireless Netw., vol. 7, no. 3, pp. 211-219, 2001.

[20] S. Pattaramalai, V. Aalo, and G. Efthymoglou, "Evaluation of call performance in cellular networks with generalized cell dwell time and callholding time distributions in the presence of channel fading," IEEE Trans. Veh. Technol., vol. 58, no. 6, pp. 3002-3013, Jul. 2009.

[21] E. Del Re, R. Fantacci, and G. Giambene, "Efficient dynamic channel allocation techniques with handover queuing for mobile satellite networks," IEEE J. Sel. Areas Commun., vol. 13, no. 2, pp. 397-405, Feb. 1995.

[22] Y.-B. Lin, S. Mohan, and A. Noerpel, "Queueing priority channel assignment strategies for PCS hand-off and initial access," IEEE Trans. Veh. Technol., vol. 43, no. 3, pp. 704-712, Aug. 1994.

[23] C. H. Yoon and C. Un, "Performance of personal portable radio telephone systems with and without guard channels," IEEE J. Sel. Areas Commun., vol. 11, no. 6, pp. 911-917, Aug. 1993.

[24] S. Thajchayapong and O. K. Tonguz, "Performance implications of Pareto-distributed cell residual time in Distributed Admission Control Scheme (DACS)," in Proc. IEEE WCNC, 2005, vol. 4, pp. 2387-2392.

[25] H. Hidaka, K. Saitoh, N. Shinagawa, and T. Kobayashi, "Self-similarity in cell dwell time caused by terminal motion and its effects on teletraffic of cellular communication networks," IEICE Trans. Fundam. Electron. Commun. Comput. Sci., vol. 85, no. 7, pp. 1445-1453, 2002.

[26] K. K. Leung, W. Massey, and W. Whitt, "Traffic models for wireless communication networks," IEEE J. Sel. Areas Commun., vol. 12, no. 8, pp. 1353-1364, Jun. 1994.

[27] C. S. Bontu and E. Illidge, "DRX mechanism for power saving in LTE," IEEE Commun. Mag., vol. 47, no. 6, pp. 48-55, Jun. 2009.

[28] A. L. E. Corral-Ruizl, F. A. Cruz-Pérez, and G. Hernandez-Valdez, "Channel holding time in mobile cellular networks with generalized Coxian distributed cell dwell time," in Proc. IEEE PIMRC, 2010, pp. 2348-2353.

[29] A. L. E. Corral-Ruiz, F. A. Cruz-Perez, and G. Hernandez-Valdez, "Cell dwell time and channel holding time relationship in mobile cellular networks," in Wireless Communications and Networks-Recent Advances, A. Eksim, Ed. Rijeka, Croatia: InTech, 2012.

[30] Evolved Universal Terrestrial Radio Access (E-UTRA) and Evolved Universal Terrestrial Radio Access network (E-UTRAN): Overall Description, 3GPP TS 36.300, Release 11. 3rd Generation Partnership Project, Sophia Antipolis Cedex, France, 2011. 
[31] J. Huang, F. Qian, A. Gerber, Z. M. Mao, S. Sen, and O. Spatscheck, "A close examination of performance and power characteristics of $4 \mathrm{G}$ LTE networks," in Proc. ACM MobiSys, 2012, pp. 225-238.

[32] L. Kleinrock, Queueing Systems: Theory. New York, NY, USA: Wiley, 1979.

[33] E. D. Re, R. Fantacci, and G. Giambene, "Efficient dynamic channel allocation techniques with handover queueing for mobile satellite networks," IEEE J. Sel. Areas Commun., vol. 13, no. 2, pp. 704-712, Feb. 1994.

[34] S. Sae-ung and S. Lertprapai, "A comparision of scale parameter estimators in the 2-parameter exponential distribution based on multiple criteria decision making," Thai J. Math., vol. 8, no. 4, pp. 73-82, 2010.

[35] J. Medhi, Stochastic Models in Queueing Theory. New York, NY, USA: Academic, 1991.

[36] P. V. Orlik and S. S. Rappaport, "A model for teletraffic performance and channel holding time characterization in wireless cellular communication," in Proc. Universal Pers. Commun., 1997, pp. 671-675.

[37] W. Li and A. S. Alfa, "Channel reservation for handoff calls in a PCS network," IEEE Trans. Veh. Technol., vol. 49, no. 1, pp. 95-104, Jan. 2000.

[38] T. K. Christensen, B. F. Nielsen, and V. B. Iversen, "Phase-type models of channel-holding times in cellular communication systems," IEEE Trans. Veh. Technol., vol. 53, no. 3, pp. 725-733, May 2004.

[39] J. L. Schiff, The Laplace Transform-Theory and Applications. New York, NY, USA: Springer-Verlag, 1999.

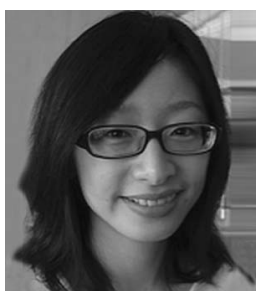

Bying-He Ku received the Ph.D. degree in computer science from National Tsing Hua University, Hsinchu, Taiwan, in 2010.

Since 2012, she has been a Postdoctoral Researcher with the Institute of Information Science, Academia Sinica, Taipei, Taiwan. Her current research interests include wireless sensor networks, ad hoc networks, Long-Term Evolution, softwaredefined networks, and information security.

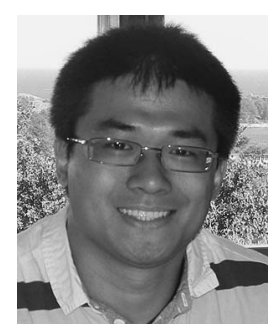

Y1 Ken (S'08-M'13) received the Ph.D. degree in information communication and technology from the University of Agder, Kristiansand, Norway, in 2012.

Since 2012, he has been an Assistant Researcher with National Chiao Tung University, Hsinchu, Taiwan. His current research interests include security and performance analysis in wireless sensor networks, ad hoc networks, and mesh networks, Long-Term Evolution, smart grids, and e-health security.

Dr. Ren received the Best Paper Award at the 2012 IEEE International Conference on Mobile Data Management.

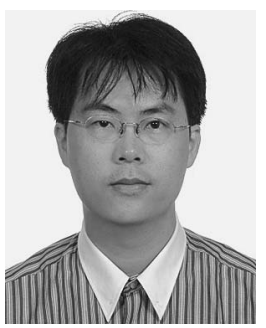

Jeng-Feng Weng (S'03-M'12) received the B.S degree from the Department of Industrial Technology Education, National Kaohsiung Normal University (NKNU), Kaohsiung, Taiwan, R.O.C., in 1997, the M.S. degree from the Department of Computer Science, National Chiao Tung University (NCTU), Hsinchu, Taiwan, R.O.C., in 2002, and the Ph.D degree from the Department of Computer Science, National Tsing Hua University (NTHU), Hsinchu, Taiwan, R.O.C., in 2012

$\mathrm{He}$ is currently an Adjunct Assistant Professor with the Department of Finance, National United University, Miaoli, Taiwan. His research interests include wireless multicast, security, key management, and performance evaluation.

Dr. Weng served as a Technical Program Committee Member for IEEE conferences, including the IEEE Symposium on Wireless Technology and Applications, the IEEE Symposium on Industrial Electronics and Applications, the IEEE International Conference on Power and Energy, the IEEE AsiaPacific Conference on Applied Electromagnetics, and the IEEE International Conference on Engineering Education.

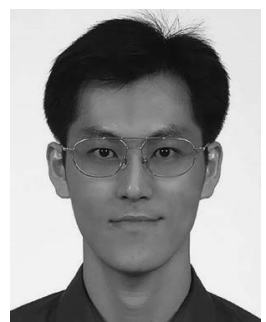

Jyh-Cheng Chen (S'96-M'99-SM'04-F'12) received the Ph.D. degree from the State University of New York at Buffalo, Buffalo, NY, USA, in 1998

He was a Research Scientist with Bellcore/ Telcordia Technologies, Morristown, NJ, USA, from 1998 to 2001 and a Senior Scientist with Telcordia Technologies, Piscataway, NJ, from 2008 to 2010. From 2001 to 2008, he was with the Department of Computer Science, National Tsing Hua University (NTHU), Hsinchu, Taiwan, as an Assistant Professor, as an Associate Professor, and as a Professor From 2011 to 2014, he was also the Director of the Institute of Network Engineering with National Chiao Tung University (NCTU), Hsinchu. Since 2010, he has been a Faculty Member and is currently a Distinguished Professor with the Department of Computer Science, NCTU. He is also serving as a Convener for the Computer Science Program of the Ministry of Science and Technology, Taiwan.

Dr. Chen received numerous awards, including the Outstanding Teaching Award from the College of Computer Science, NCTU, the Outstanding I. T. Elite Award, the Mentor of Merit Award from NCTU, the K. T. Li Breakthrough Award from the Institute of Information and Computing Machinery, the Outstanding Professor of Electrical Engineering Award from the Chinese Institute of Electrical Engineering, the Outstanding Research Award from the Ministry of Science and Technology, the Outstanding Teaching Award from NTHU, the Best Paper Award for Young Scholars from the IEEE Communications Society Taipei and Tainan Chapters and the IEEE Information Theory Society Taipei Chapter, and the Telcordia CEO Award. He is a Distinguished Member of the Association for Computing Machinery. He is a member of the Fellows Evaluation Committee of the IEEE Computer Society in 2012 and 2016.

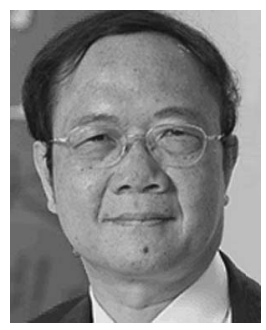

Wen-Tsuen Chen (F'94) received the B.S. degree in nuclear engineering from National Tsing Hua University, Hsinchu, Taiwan, in 1970 and the M.S. and $\mathrm{Ph} . \mathrm{D}$. degrees in electrical engineering and computer sciences from the University of California, Berkeley, Berkeley, CA, USA, in 1973 and 1976, respectively.

Since 1976, he has been with the Department of Computer Science, National Tsing Hua University, where he served as the Chairman of the Department, the Dean of the College of Electrical Engineering and Computer Science, and the President of National Tsing Hua University. In March 2012, he joined Academia Sinica, Taipei, Taiwan, as a Distinguished Research Fellow of the Institute of Information Science with joint appointment as a Sun Yun-suan Chair Professor of National Tsing Hua University. His research interests include computer networks, wireless sensor networks, mobile computing, and parallel computing.

Dr. Chen received numerous awards for his academic accomplishments in computer networking and parallel processing, including the Outstanding Research Award from the National Science Council, the Academic Award in Engineering from the Ministry of Education, the Technical Achievement Award, and the Taylor L. Booth Education Award from the IEEE Computer Society. He is currently a lifelong National Chair of the Ministry of Education, Taiwan. He is also the Founding General Chair of the IEEE International Conference on Parallel and Distributed Systems and the General Chair of the 2000 IEEE International Conference on Distributed Computing Systems, among others. He is a Fellow of the Chinese Technology Management Association. 